\title{
An Innovative Hybrid Algorithm for Solving Combined Economic and Emission Dispatch Problems
}

\author{
Pooja Verma ( $\nabla$ poojatwri28@gmail.com ) \\ Indira Gandhi National Tribal University \\ Raghav Prasad Parouha \\ Indira Gandhi National Tribal University
}

\section{Research Article}

Keywords: Economic load dispatch, Combined economic emission dispatch problem, Meta-heuristic algorithms, Hybrid algorithm

Posted Date: February 23rd, 2022

DOI: https://doi.org/10.21203/rs.3.rs-1376881/v1

License: (c) (i) This work is licensed under a Creative Commons Attribution 4.0 International License.

Read Full License 


\section{An innovative hybrid algorithm for solving combined economic and emission dispatch problems}

*Pooja Verma, Department of Mathematics, Indira Gandhi National Tribal University, Amarkantak, M.P., India; Email: poojatwri28@ gmail.com Raghav Prasad Parouha, Department of Mathematics, Indira Gandhi National Tribal University, Amarkantak, M.P., India; Email: rparouha@ gmail.com *Corresponding author

Abstract: As environmental concerns have grown, the combined economic emission dispatch (CEED) problem has gotten a lot of attention. Both the cost of fuel and the emission pollution caused by it must be kept to a minimum. As a result, this paper presents an innovative hybrid approach (ihPSODE) for solving CEED problems. This hybrid technique incorporated novel differential evolution (nDE) and particle swarm optimization (nPSO). Where nDE introduces a new mutation approach and crossover rate (to prevent premature convergence) as well as nPSO introduces a new acceleration coefficient, inertia weight and position improve equation (to alleviate the stagnation). So as to balance among local and global search ability, after ihPSODE population evaluation, the best half individuals are determined and the rest individuals are discarded. Then, nPSO is used in the current population (to sustain exploration and exploitation) and $\mathrm{nDE}$ is employed in the nPSO generated population (to improve convergence accuracy). The competence of the proposed algorithms (ihPSODE, nPSO and $\mathrm{nDE}$ ) are inspected on 23 unconstrained benchmark function and then solved 3 test system (3-, 6- and 40-unit) of economic load dispatch (ELD) and 3 test system (3-, 10- and 40-unit) of CEED problem. The experiments have denoted that the proposed algorithms show competitive results and significant performances.

Keywords: Economic load dispatch, Combined economic emission dispatch problem, Meta-heuristic algorithms, Hybrid algorithm.

\section{Introduction}

In modern power system operation, economic load dispatch (ELD) is a critical optimization problem (Mansor et al. 2018). The basic goal of the ELD problem is to lower total generation costs while keeping load demand and other equality and inequality limitations in mind. Apart from the producing capacity limits, the classic ELD primarily analyses the power balance constraint. But, due to practical limitations in power system operation, ELD essentially consider a multiplicity of real-world constraints like transmission loss, prohibited operating zones, multi-fuel options, ramp rate limits, spinning reserve along with system power demand etc. It resulted with a non-convex nonlinear ELD problem and finding an optimum solution of this type problem is very challenging and time-consuming. The environmental constraint, which consists of Carbon oxides (Cox), Nitrogen oxides (NOx) and Sulphur oxides (Sox), infects the air, is one of those limitations that is always taken into account. By properly allocating load among available generators, the hazardous environmental pollutants released by fossil-fuel power plants can be decreased. However, the power plant's operational costs would rise. As a result, a solution must be found that balances both emissions and fuel costs. It can have been attained by 'combined economic and emission dispatch (CEED)' problem. The key goal of the CEED problem is to concurrently reduce fuel costs and emissions while meeting equality and inequality limits plus load demand. Mathematically, the ELD and CEED problem can be expressed as an optimization (minimization) problem, as shown below.

\subsection{Mathematical problem formulation}

\section{Economic load dispatch (ELD)}

In ELD the total fuel cost $(\$ / \mathrm{hr})$ can be mathematical expressed as below:

$$
F_{t}=\sum_{i=1}^{n}\left(a_{i} P_{i}^{2}+b_{i} P_{i}+c_{i}\right)
$$

Also, in view of valve-point loadings effect the fuel cost function given as follows.

$$
F_{t}=\sum_{i=1}^{n}\left[\left(a_{i}+b_{i} P_{i}+c_{i} P_{i}^{2}\right)+\left|e_{i} \sin \left(f_{i}\left(P_{i}^{\min }-P_{i}\right)\right)\right|\right]
$$

where $F_{t}$ : total fuel cost of generations $(\$ / \mathrm{hr}) ; a_{i}, b_{i}$ and $c_{i}$ : cost factors of a generator $i ; e_{i}$ and $f_{i}$ : fuel cost quantities for valve point effects of a generator $i$; $P_{i}$ : power output of the $i^{\text {th }}$ generator. 
The constraints of ELD problems are listed as follows.

○ Generator limits

$$
P_{i}^{\min } \leq P_{i} \leq P_{i}^{\max }
$$

where $P_{i}^{\min }$ (minimum) and $P_{i}^{\max }$ (maximum) power generation by unit $i$.

- Power balance

$$
\begin{gathered}
\left.\sum_{i=1}^{n} P_{i}=P_{D}(\text { total load demand })+P_{L} \text { (total transmission line loss }\right) \\
P_{L}=\sum_{i=1}^{n} \sum_{j=1}^{n} P_{i} B_{i j} P_{j}+\sum_{i=1}^{n} P_{i} B_{o i}+B_{o o}
\end{gathered}
$$

where $B_{i j}, B_{o i}$ and $B_{o o}$ are transmission loss coefficient.

○ Prohibited operating zone

$$
P_{i}^{\min } \leq P_{i} \leq P_{i, 1}^{l}: P_{i, k-1}^{u} \leq P_{i} \leq P_{i, k}^{l}: P_{i, n_{i}}^{u} \leq P_{i} \leq P_{i}^{\max } ; k=2,3, \ldots n_{i}
$$

where $n_{i}$ : number of prohibited operating zone and $P_{i, k}^{l} \& P_{i, k}^{u}$ : lower and upper limit of $k^{\text {th }}$ prohibited zone of generating unit $i$.

○ Ramp rate limit

$$
\max \left(P_{i}^{\min }, P_{i}^{t-1}-\mathrm{DR}_{i}\right) \leq P_{i}^{t} \leq \min \left(P_{i}^{\max }, P_{i}^{t-1}+\mathrm{UR}_{i}\right)
$$

where $P_{i}^{t} \& P_{i}^{t-1}$ current $\&$ previous output power and $U R_{i} \& D R_{i}$ : up \& down ramp limit of generating unit $i$.

\section{Combined economic emission dispatch (CEED)}

When generator units burn fossil fuels, pollutants such as SOx, NOx, and COx are released into the atmosphere. The overall emission of these pollutants, known as emission constrained dispatch (ECD), can be written as.

$$
E_{t}=\sum_{i=1}^{n}[\underbrace{10^{-2}\left(\alpha_{i} P_{i}^{2}+\beta_{i} P_{i}+\gamma_{i}\right)}_{\text {quadratic function }}+\underbrace{\psi_{i} \exp \left(\lambda_{i} P_{i}\right)}_{\text {exponential function }}]
$$

where $E_{t}$ : total amount of emissions ( $\left.\mathrm{lb} / \mathrm{hr}\right)$ and $\alpha_{i}, \beta_{i}, \gamma_{i}, \psi_{i} \lambda_{i}$ : emission coefficients of the $i^{\text {th }}$ unit. Moreover, simultaneously minimizing two objective function $F_{t}$ and $E_{t}$ is the main target of the CEED problem. By a price penalty factor $(h)$ methodology this bi-objective problem can be transformed into a single objective problem as follows.

$$
\varphi_{t}=F_{t}+h \times E_{t}
$$

where $\varphi_{t}$; total cost of the system operation. The price penalty factor $h$ can be calculated by the following procedures for a particular load demand.

(i). Calculate the ratio $\frac{F_{t}\left(P_{i}^{\max }\right)}{E_{t}\left(P_{i}^{\max }\right)}=h_{i}, i=2,3, \ldots n \$ / \mathrm{kg}$.

(ii). Sort the obtained $h_{i}$ values in ascending order.

(iii). Add $P_{i}^{\max }$ of each unit one at a time starting from the unit with smallest $h_{i}$ until $\sum P_{i}^{\max } \geq P_{D}$.

(iv). Catch the last value of $h_{i}$ that attains the previous situation which signifies the price penalty factor for the given load.

Equation (5) can be modified as follow (for providing a balance between minimization of the fuel cost and emission).

$$
\varphi_{t}=w \times F_{t}+(1-w) \times h \times E_{t}
$$

where $w$ (specifies type of the optimization problem) is the weight factor and if -

(i). $\quad w=1$ infers ELD problem

(ii). $\quad w=0$ implies ECD problem

(iii). $\quad w=0.5$ indicates CEED problem 


\subsection{Related literature survey}

To handle engineering optimization challenges, many traditional optimization techniques such as Newton and quasi-Newton have been created. Moreover, they have some intrinsic limitations, such as high computing cost, local optimal stagnation, and search space derivation (Simpson et al. 1994). It's also challenging to locate the best solution during the problem-solving process. To circumvent the limitations of traditional optimization approaches, meta-heuristics algorithms (MAs) have been developed to handle complicated engineering optimization problems. The MAs can be separated into 4 sets according to the mechanical variances as-

\begin{tabular}{|c|c|c|}
\hline MAs & Motivated from & Example \\
\hline $\begin{array}{l}\text { Evolutionary algorithms } \\
\text { (EAs) }\end{array}$ & biology & $\begin{array}{l}\text { genetic algorithm (GA) (Davis 1991), differential } \\
\text { evolution (DE) (Storn and Price 1997), } \\
\text { Backtracking Search Optimization Algorithm (BSA) } \\
\text { (Civicioglu P 2013; Hassan and Rashid 2019a; } \\
\text { Hassan and Rashid 2019a) etc. }\end{array}$ \\
\hline $\begin{array}{l}\text { Swarm } \\
\text { algorithms } \\
\text { (SIAs) }\end{array}$ & social bugs & $\begin{array}{l}\text { particle swarm optimization (PSO) (Kennedy and } \\
\text { Eberhart 1995), artificial bee colony (ABC) } \\
\text { (Karaboga and Basturk 2007), cuckoo search (CS) } \\
\text { (Yang and Deb 2009), krill herd (KH) (Gandomi and } \\
\text { Alavi 2012) grey wolf optimizer(GWO) (Mirjalili et } \\
\text { al. 2014) dragonfly algorithm (DA) (Mirjalili 2016), } \\
\text { harris hawks optimization (HHO) (Heidari et al. } \\
\text { 2019) etc. }\end{array}$ \\
\hline $\begin{array}{l}\text { Physics-based algorithms } \\
\text { (PBAs) }\end{array}$ & phenomenon & $\begin{array}{l}\text { harmony search (HS) (Geem et al. 2001), } \\
\text { gravitational search algorithm (GSA) (Rashedi et al. } \\
\text { 2009), water cycle algorithm (WCA) (Eskandar et } \\
\text { al. 2012), equilibrium optimizer (EO) (Faramarzi et } \\
\text { al. 2019) etc. }\end{array}$ \\
\hline $\begin{array}{l}\text { Human behaviour based } \\
\text { algorithms } \\
\text { (HBAs) }\end{array}$ & nuा & $\begin{array}{l}\text { teaching-learning-based optimization (TLBO) (Rao } \\
\text { et al. 2011), mine blast algorithm (MBA) (Sadollah } \\
\text { et al. 2013) etc. }\end{array}$ \\
\hline
\end{tabular}

The employment of the "trial-and-error" method in looking for solutions is the fundamental advantage of these algorithms. As a result, these methods have been successfully used to global optimization issues. Between popular MAs, PSO and DE successfully applied in diverse optimization parts due to their dominant search ability and simple arrangement. However, various drawbacks of DE and PSO limit their application in complex optimization contexts.

The fundamental drawback of PSO is that it can quickly become trapped in a local optimal solution zone. As a result, in PSO, speeding convergence speed and avoiding local optimal solutions are two essential challenges. To address such concerns, many potential changes to the PSO have been proposed in recent literature. Espitia and Sofrony (2018) proposed VPSO in which particle vortex behavior and particle circular motions are implemented for improving search capacity and escaping the local minima respectively. To find the optimum of the current search and gradually explore the search space, Yu et al. (2018) proposed SHPSO by the implementation of social learning PSO. Chen et al. (2018) devised PSOCO where two distinct crossover operations are taken to produce promising exemplars in order to balance diversity. A self-adaptive tool and unique factor $\left(w, \mathrm{c}_{1}, \mathrm{c}_{2}\right)$ utilized for enhancing each particle position depending on their fitness in UAPSO which is devised by Isiet and Gadala (2019). Hosseini et al. (2019) developed HAFPSO where fractional-order derivatives and hunter-attack strategy are used to accelerate convergence and avoid stagnation respectively. Khajeh et al. (2019) proposed MPSO where a novel particle initializing scheme with random distribution is used for uniformly covering the search space. Ang et al. (2019) invented CMPSOWV, in which two diversity maintenance schemes (multiswarm technique and probabilistic mutation operator) are used to prevent the premature convergence. Lanlan et al. (2020) proposed NOPSO where non-inertial velocities update formula, opposition based learning strategy and adaptive elite mutation strategies are employed to avoid local optimum trapping. 
Xiong et al. (2020) proposed NMSPSO where three strategies- novel information exchange strategy (for information transfer between sub-swarms), novel leaning strategy (for speed up the convergence) and novel mutation strategy (for better exploration) are incorporated. In the ground of real-world problems, DE also has noteworthy performance and become a great optimizer. Still, it has few concerns like local exploitation ability and convergence rate. So as to reduce its concerns, hordes of effective and robust DE has been intended in the literature. Qiu et al. (2018) proposed MMDE where a novel bottom-boosting mechanism (to maintain the reliability), partial-regeneration strategy (to identified the promising solutions) and mutation operator DE/current/1 (to explore over solution space) are introduced. Zhang and Li (2018) developed DEPS in which a modified parent selection scheme is chosen to use the experience of successful parents while selecting them in mutation operator. Huang et al. (2018) invented hypercube-based NCDE where hypercube neighbourhood based mutation (to maintain the neighborhood size in a reasonable range) and self-adaptive techniques (to control the hypercube's radius vector) are used. Yang et al. (2019) developed daDE in which a modified mutation rule is created to utilize the information of the current and the former individual's altogether. It has great benefits on the robustness and convergence speed. Liu et al. (2019) proposed HDEMCO where two layers' top layer (where multi cross operation perform that provides rapid convergence) and bottom layer (where success-historybased adaptive DE is implemented for better global search) are considered. Gui et al. (2019) devised MRDE in which different trial vector generation strategies, regroup scheme and an adaptive strategy are performed to speed up the convergence rate. Li et al. (2019) developed EJADE where a sorting mechanism and a dynamic population reduction strategy are employed to speed up the convergence rate and maintain the diversity respectively. Hu et al. (2020) invented BADE where an annealing strategy allow the searching space to explore and accelerate the convergence too. Ben (2020) proposed ADE where initial population and a new difference vector (in mutation phase) are created by the knowledge of damage scenario structure and dispersion of individuals respectively.

Moreover, to increase the performance of a single algorithm, one of the primary research advices is the hybrid strategy. Because of diverse optimization methods have dissimilar search behaviours and benefits. Hence, to reduce their individual weaknesses (like premature convergence, stacks at local optima etc.), hybrid methods are now preferred more to solve complex optimization problems. As a result, many hybrid algorithms for DE and PSO are presented in the literature in order to improve their performance. Seyedmahmoudian et al. (2015) proposed DEPSO, where DE is employed to adds diversity on traditional PSO. However, it may not appropriate for multimodal optimization problems. Parouha and Das (2015) devised DPD in which DE is executed in the inferior and superior groups, while PSO is employed in the mid-group. But, for solving complex real-world problems it may need some moderations. Tang et al. (2016) proposed HNTVPSO-RBSADE, which employs a nonlinear time varying PSO and a ranking based self-adaptive DE to result in dynamic exploration and exploitation. Parouha and Das (2016a) developed MBDE in which swarm mutation and swarm crossover for DE is used to direct knowledge and improve the solution quality. Parouha and Das (2016b) proposed DEPSO-DE in which the population is divided into three groups $(\mathrm{A}, \mathrm{B}, \& \mathrm{C})$ and executed in parallel manner. Famelis et al. (2017) devised DE-PSO where DE is merged with a velocity-update rule of PSO to enhance diversity. Mao et al. (2018) proposed DEMPSO in which DE is added first to lessen the search space and then acquired populations used modified PSO (MPSO) as an initial population to speed up the convergence rate. Tang et al. (2018) developed SAPSO - mSADE in which self-adaptive PSO (SAPSO) and modified self-adaptive DE (mSADE) are evolved to balance diversity and reduce potential stagnation respectively. Too et al. (2019) invented BPSODE where the strength of binary PSO (BPSO) and binary DE (BDE) are inherited and computed in sequence. Dash et al. (2020) proposed HDEPSO in which three DE operations (mutation, modified crossover and selection) are fused with the best particles of PSO for enhancing global searching ability. Parouha and Verma (2021) proposed innovative hybrid algorithm of DE and PSO for bound-unconstrained optimization and ELD problem with or without valve point effects. It integrated with novel PSO (to escape stagnation) and DE (to avoid premature convergence). Further, Verma and Parouha (2021) applied the innovative hybrid algorithm to solve non-convex dynamic economic dispatch problem and numerical, graphical as well as comparative results shows its capability to solve considered optimization problems.

Moreover, a related recent review of DE, PSO and their hybrids as well as other MAs variants for solving CEED problem are mentioned as further. Mahdi et al. (2018) proposed QBA, in which a cubic criterion function is employed to represent this problem to reduce the nonlinearities of the system. The 
addition of quantum behaviour in bats, which eventually contributes to the diversification of population and diversifies the foraging habitats. In addition, early convergence in BA can be prevented. Jiang et al. (2019) devised GPSOA, where it integrates PSO with gravitation laws of GSA. Here, the particle's velocity is reorganized by random support of PSO and GSA. Additionally, Weibull-based probability density function is also used, to designate the stochastic individualities of wind speed and output power. Rezaie et al. (2019) proposed CIHSA, which is the combination of IHSA and CHSA. Where IHSA has a suitable convergence rate with high accuracy and CHSA has a high strength to find the best solutions in altered runs. Moreover, to dynamically tuning the parameters, employing virtual harmony memories and generate random numbers it uses chaotic patterns. Goudarzi et al. (2019) proposed MGAIPSO, it utilizes three operators, an arithmetic crossover and a mutation operator from GA to produce elite off-springs and maintain diversity; a non-linear time-varying double-weighted technique from PSO to obtain a substantial balance between exploration and exploitation. Edwin Selva Rex et al. (2019) proposed a novel hybrid algorithm (GA-WOA) using GA and Whale optimization for solving CEED problem. This method verified on 4 different test systems and it is superior to other heuristic methods with slight increase in the CPU execution time. Rashid et al. (2020) invented MIW-PSO, in which a multiple inertia weight is incorporated in PSO to improve its convergence characteristics for minimizing fuel cost and pollutant emission in the uncertain energy production expense and random load. Bibi et al. (2020) developed GOA, where the comfort zone operator of GOA assists in extracting stupendous simulations results of minimized fitness of multi objective functions that shows the efficiency of GOA in term of highly optimal and feasible solution satisfying all the system equality and inequality operational constraint. Khatsu et al. (2020) proposed PPSO in which a linear and non-linear time varying weight inertia factor (LWF and NLWF) are introduced in PPSO to enhance its searching ability. Goyal et al. (2020) proposed BBO, where an optimum generator scheduling has been achieved by employing BBO with all system constraints. Sakthivel et al. (2020) proposed MOSSA, it integrates squirrel search algorithm along with Pareto-dominance principle to generate non-dominated solutions. Also, it employed outward elitist depository tool with flocking distance categorization (to retain the distribution diversity) and utilized fuzzy decision-making strategy (to select the finest cooperated solution). Ajayi and Heymann, (2021) devised MSA. It is encouraged by the effort of moths the moonlight towards. Also, to provide the essential spinning reserve capacity, it slated thermal generators as solar energy is intermittent in nature. Hassan et al. (2021) proposed CAEO, which uses the chaotic maps which enhance a variety of the solution spaces and improve the convergence capabilities to achieve the optimum solutions as well as avoid the local minima.

\subsection{Research gaps (Inspiration/motivation)}

Despite the fact that a large number of MAs have been introduced in the literature, they have not been able to solve a wide range of problems (Wolpert and Macready 1997). Particularly, for some problems an algorithm can produce satisfactory outcomes but not for others. As a result, for solving a variety of optimization problems there is a necessity to develop some efficient algorithms. Furthermore, over separate efforts hybrid algorithms are now favored more to solve complex real-world problems. Hence, from the inspiration of above mentioned facts and literature investigation motivation of this study is to design an effective and innovative hybrid algorithm with the combination of novel variants of MAs. Between popular MAs, PSO and DE as well as their hybrids successfully applied in diverse optimization parts due to their leading search ability and simple structure. Consequently, after a wide analysis of the literature on diverse alternatives of PSO and DE with their hybrids the subsequent resulting opinions is evaluated and encouraged form them.

(i). The PSO is largely dependent on its parameters (which direct particles to the optimum) and position update (to balance diversity). As a result, numerous investigators have attempted to improve the accuracy and speed of PSO by modifying its control parameters and position update equation.

(ii). In DE mutation and crossover schemes with their associated control factors, are used to generate the global best solution, which is favorable for refining convergence performance. Hence, the most appropriate schemes and their related factor are regarded as a critical research study for DE.

(iii). Owing to their efficiency in solving complex real-world problems hybrid methods have caught the more interest of investigators. As per investigation, PSO and DE have balancing properties and 
their hybrids have recently gained more popularity to solve numerous real-world problems. From now, to the best of our information, figuring out how to hybridize PSO and DE is still an open research problem.

\subsection{Contribution}

Inspired by above remarks and literature study planned the following for solving CEED problems.

(i). an innovative hybrid algorithm (ihPSODE): it integrated with suggested novel PSO (to increase the population diversity) and novel DE (it produce perturbations to avoid the algorithm trapping into local optima).

(ii). novel PSO (nPSO): it involves new gradually changeable (increasing and/or decreasing) parameters and new equation for position update.

(iii). novel $\mathrm{DE}(\mathrm{nDE})$ : it includes combination of novel operators (mutation and crossover) and new related control parameters.

The proposed algorithms have validated on 23 typical unconstrained benchmark functions then used to solve 3 test systems (3, 6 and 40-unit test system) of ELD and 3 test systems (3, 10 and 40-unit test system) of CEED problem. Comparative experiments endorse the efficiency and superiority of the presented algorithms.

The rest of this paper is presented in the following. Section 2 presents the proposed algorithms in details. Proposed algorithms are tested on 23 unconstrained benchmark functions in Section 3. In Section 4, the proposed algorithms applied on 3 different test systems of ELD and CEED problems. Conclusions would be drawn in section 5 with future work.

\section{Proposed methodology}

This section briefs the basics PSO and DE then discussed and the proposed methodology in detail.

Particle Swarm Optimization (PSO) (Kennedy and Eberhart 1995)

In a $D$-dimensional optimization space $x_{i}=\left(x_{i, 1}, x_{i, 2}, \ldots, x_{i, D}\right)$ and $v_{i}=\left(v_{i, 1}, v_{i, 2}, \ldots, v_{i, D}\right)$ denote the position and velocity of the $i^{\text {th }}$ particle, respectively. During the evolutionary process, each individual constantly adjusts its velocity and position by following the personal best experience pbest $_{i}=\left(\right.$ pbest $_{i, 1}$, pbest $_{i, 2}, \ldots$, pbest $\left._{i, D}\right)$ and the population best experience gbest ${ }_{j}=$ $\left(\right.$ gbest $_{1}$, gbest $_{2}, \ldots$, gbest $\left._{D}\right)$. The specific mathematical formulations are presented as:

$$
\begin{gathered}
v_{i, j}^{t+1}=w v_{i, j}^{t}+c_{1} r_{1}\left(\text { pbest }_{i, j}^{t}-x_{i, j}^{t}\right)+c_{2} r_{2}\left(\text { gbest }_{j}^{t}-x_{i, j}^{t}\right) \\
x_{i, j}^{t+1}=x_{i, j}^{t}+v_{i, j}^{t+1}
\end{gathered}
$$

where $t$ denotes the number of iterations. $c_{1}$ and $c_{2}$ are two important control parameters called acceleration coefficients. $r_{1}$ and $r_{2}$ are two randomly selected numbers within the range [0,1]. $w$ is the inertia weight

\section{Differential Evolution (DE) (Storn and Price 1997)}

In DE algorithm, an initial population which includes $n p$ individuals are randomly generated in the $D$-dimensional solution space. In the searching space, each individual represents a candidate solution. Three main operations on DE given as follows.

(i). Mutation: After initialization, to increase the population diversity, DE hires the mutation operator to create a mutation vector (mutant individual) $v_{i, j}^{t}$ with respect to the target vector $x_{i, j}^{t}$ at generation $t$ as

$$
v_{i, j}^{t+1}=x_{r_{1}}^{t}+F\left(x_{r_{2}}^{t}-x_{r_{3}}^{t}\right)
$$

where $r_{1}, r_{2}$ and $r_{3}$ are jointly different integers taken from [1,NP] and are distinct from the index $i$. The control parameter $F$ is a scaling factor, which amplifies the difference vector.

(ii). Crossover: After the above operation, a binomial crossover which recombines the target vector $x_{i, j}^{t}$ and the mutation vector $v_{i, j}^{t+1}$, is usually applied to generate a new trail vector $u_{i, j}^{t+1}$ as follows. 


$$
u_{i, j}^{t+1}=\left\{\begin{array}{l}
v_{i, j}^{t+1} ; \text { if } \operatorname{rand}(j) \leq C_{r} \text { or } j=\operatorname{rnbr}(i) \\
x_{i, j}^{t} ; \text { if } \operatorname{rand}(j)>C_{r} \text { or } j \neq \operatorname{rnbr}(i)
\end{array}\right.
$$

where $j=1, \ldots, D \cdot \operatorname{rand}(j)$ is a random number on the interval $[0,1]$. Crossover rate $C_{r}$ is another control parameter in DE algorithm. To ensure that $u_{i, j}^{t+1}$ get at least one parameter from $v_{i, j}^{t+1}$, $\operatorname{rnbr}(i)$ is a randomly selected integer within $[1, D]$.

(iii). Selection: following the crossover operation, DE applies a greedy strategy to select a vector for the next generation as follows. If the trial vector $u_{i, j}^{t+1}$ has better or equal fitness, it will be retained in the next generation. Otherwise, the target vector $x_{i, j}^{t}$ will survive.

$$
x_{i, j}^{t+1}=\left\{\begin{array}{l}
u_{i, j}^{t+1} ; \text { if } f\left(u_{i, j}^{t+1}\right) \leq f\left(x_{i, j}^{t}\right) \\
x_{i, j}^{t} ; \text { Otherwise }
\end{array}\right.
$$

\section{1 novel PSO (nPSO)}

The specific operation of nPSO may be separated as below steps.

1-step Generate initial population $x_{i}^{t}=\left\{x_{i, 1}^{t}, x_{i, 2}^{t}, \ldots, x_{i, D}^{t}\right\} i=1,2, \ldots, n p$, randomly follows the uniform distribution.

2-step Compute the objective function value $f\left(x_{i}^{t}\right)$.

3-step pbest and gbest selection with updating.

4-step Each individual adjusts its velocity and position as follows-

As per earlier investigation in PSO-

(i). small and large $w$ values effect exploration and exploitation in earlier and latter stages correspondingly.

(ii). acceleration coefficient $c_{1}$ (decreasing) and $c_{2}$ (increasing) supports personal and global best results individually.

(iii). accuracy of achieved solution is not high and particles oscillate near local optimum for next iterations as good situation can be used in positions update procedure.

Encouraged by above statistic in nPSO following parameter suggested.

(i). a linearly decreased $w\left(w=\frac{1}{2}\left(1+\frac{t_{\max }-t}{t_{\max }}\right)\right)$.

(ii). gradually decreased $c_{1}\left(c_{1}=\left(\frac{1}{1+\frac{t}{t_{\max }}}\right)\right)$ and gradually increased $c_{2}$ $\left(c_{2}=\left(\frac{1}{2-\frac{t}{t_{\max }}}\right)\right)$ acceleration factor.

(iii). $n_{t}=e^{\left(1-\frac{t_{\max }+t}{t_{\max }-t}\right)}$ (a non-linear decreasing factor), where $t \& t_{\max }$ is current iterations \& maximum number of iterations respectively, introduced in position update equation, it may have benefitted once nPSO executes local search in later iterations as particles get nearby to the global best result.

The offered factor behavior illustrated in Fig. 1 \& Fig. 2.

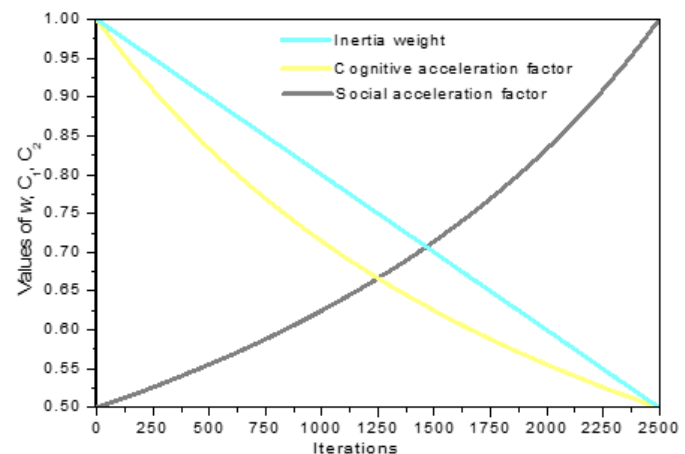

Fig. $1 w, c_{1} \& c_{2}$ behavior during evolution process $c_{1}$

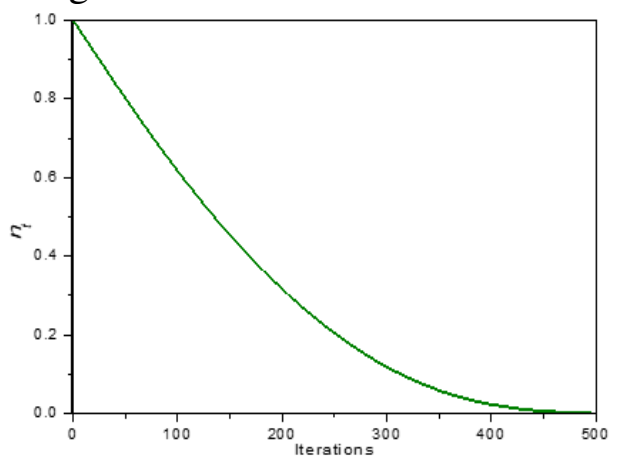

Fig. 2 Factor $n_{t}$ behavior during evolution process 
Under the above circumstance, eq. (6)- to update velocity and eq. (7)- to update position can be replaced as follows.

$$
\begin{gathered}
v_{i}^{t+1}=\left(\frac{1}{2}\left(1+\frac{t_{\max }-t}{t_{\max }}\right)\right) v_{i}^{t}+\left(\frac{1}{1+\frac{t}{t_{\max }}}\right) r_{1}\left(\text { pbest } t_{i}^{t}-x_{i}^{t}\right)+\left(\frac{1}{2-\frac{t}{t_{\max }}}\right) r_{2}\left(\text { gbes }-x_{i}^{t}\right) \\
x_{i}^{t+1}=x_{i}^{t}+n_{t} v_{i}^{t+1}
\end{gathered}
$$

5-step Stop if reaching the convergence/termination condition otherwise repeat the steps from 2.

\section{2 novel DE (nDE)}

The specific operation of $\mathrm{nDE}$ may be divided into the following stages.

1-step In the $D$ - dimensional solution space, an initial population generated uniform randomly which includes $n p$ individuals.

2-step Compute the objective function value.

3-step (i). Mutation

Mathematically, the proposed mutation strategy can be described as follows.

$$
v_{i, j}^{t+1}=x_{i, j}^{t}+\tau \times \operatorname{rand}(0,1) \times\left(\text { best }_{i, j}^{t}-x_{i, j}^{t}\right)
$$

where $v_{i}^{t}$ : mutant vector, $x_{i}^{t}$ - target vector, $\tau$ : convergence parameter, $\operatorname{rand}(0,1)$ : uniformly scattered random number among $0 \& 1$ and best $_{i}$ : best vector.

In eq. (13), importance of $\tau$ has an significant influence on presentation of $\mathrm{nDE} \&$ i $h$ PSODE (defined below). The vibrant amendments of $\tau$ are specified as below and its behavior depicted in Fig. 3 as early, middle and later stages on evolution procedure.

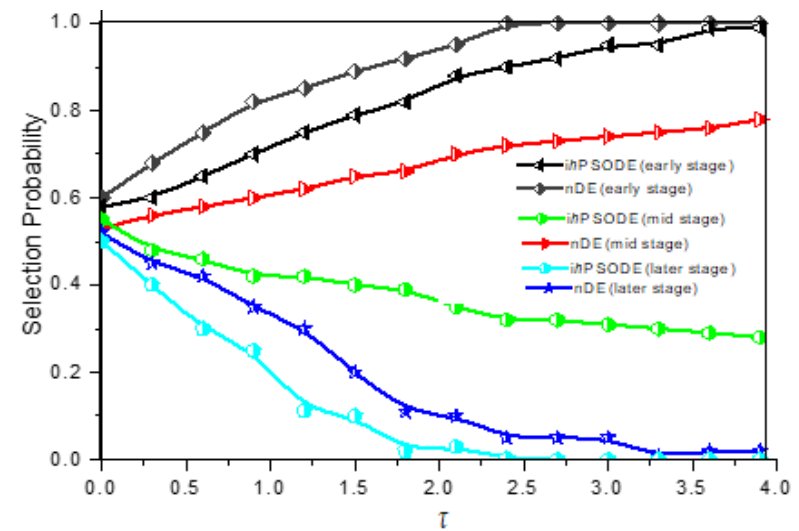

Fig. 3 Factor $\tau$ behavior during evolution process

it may observe that from Fig. 3 during the evolution following stages.

\begin{tabular}{l|l|l}
\hline early & middle & later \\
\hline the choice probability of & the choice probability & the choice probability of \\
mutation scheme is nearer & befits minor, which is not & ihPSODE and nDE would \\
to $100 \%$ and population & helpful for both to & be improved when $\tau \leq$ \\
diversity can be sustained & converge and hence $\tau$ & 2.2 as ihPSODE and nDE \\
effectually when $\tau \geq 1.5$. & would not be excessively & can concentrate on \\
Thus, as far as possible & large $\tau<2.5)$ Thus, & improving local \\
ihPSODE and nDE is & ihPSODE and nDE can & exploitation facility, \\
capable to examine & accelerate search while & convergence speed and \\
additional promising \\
regions.
\end{tabular}

As all, a smaller value of $\tau$ is helpful for global convergence ability as well as a larger value of $\tau$ is useful to retain population diversity. Therefore, advised to take the value of $\tau$ in between or equal to $1.5 \& 2.2$ which balance local exploitation and global exploration.

(ii). Crossover

it can be observed that from eq. (9) if $C_{r}$ becomes with following values 


\begin{tabular}{l|l}
\hline larger & smaller \\
\hline$v_{i}^{t}$ (mutation vector) pays extra to the $u_{i}^{t}$ & $x_{i}^{t}$ donates more to the $u_{i}^{t}$, it is helpful to \\
(trial vector), it is useful to speed up local & preserve global search ability and \\
search and convergence rate, but it lose & population diversity, but it makes slow \\
those target vectors $\left(x_{i}^{t}\right)$ which have & $\begin{array}{l}\text { searching process and not able to } \\
\text { generate new individual structures. }\end{array}$ \\
superior fitness. &
\end{tabular}

Hence, proposed novel $C_{r}=0.9-e^{\left(1-\frac{t_{\max }}{t_{\max }-t+1}\right)} \times 0.7$ is use in nDE to overcome the above issues. It can maintain population diversity and accelerate convergence of the nDE. Ultimately, $90 \%$ trial vectors can be created by mutation operators if $C_{r}$ is equal to 0.9 along with as crossover probability increases it may upsurge mutation degree of vectors.

(iii). Selection

Following the crossover operation, nDE applies a greedy strategy eq. (10) to select a vector for the next generation.

4-step Stop if reaching the convergence/termination condition otherwise repeats the steps from 2.

\section{3 innovative hybrid PSODE (ihPSODE)}

Primarily, $\mathrm{i} h \mathrm{PSODE}$ is created on involving higher competence of the contributed nPSO and nDE. The specific operation of $i$ hDEPSO can be revealed as below stages.

1-step In the $D$-dimensional solution space, an initial population generated uniform randomly which includes $n p$ individuals.

2-step Compute the objective function value.

3-step Sort population according to according to their performance as well as recognize best half and remove the rest population.

4-step Apply nPSO

5-step Apply nDE (in offspring generated by nPSO)

6-step Merge the population produced by $\mathrm{nPSO}$ and $\mathrm{nDE}$

7-step Stop if reaching the termination condition otherwise repeats the steps from 2.

\section{Validation of presented algorithms}

To confirm the performance of the presented algorithms, experiments are investigated on twenty-one classical unconstrained benchmark functions ( cubf $_{\mathrm{s}}$ ). Among these functions, $f_{1} \sim f_{7}, f_{8} \sim f_{13}$ and $f_{14} \sim f_{23}$ are unimodal, multimodal and fixed-dimension benchmark functions, respectively. The data of these cubf $_{\mathrm{s}}$ are scheduled in Table 1. Simulations were piloted on Intel (R) Core (TM) i5-2350, CPU @ $2.30 \mathrm{GHz}$ with $4 \mathrm{~GB}$ RAM and simulated in C language (C-free Standard 4.0). Furthermore, to handle constraint, a penalty term is added to the objective function. Because of its higher efficiency, the bracket operator penalty (Deb 1995) was chosen for this study. Besides after several tryouts fine-tuning value of $\mathrm{R}=1 e^{03}$ is recommended for presented algorithms. In each table, overall best values are emphasized with bold of corresponding algorithms.

The produced result by proposed algorithms on 23 cubfs is compared with traditional algorithms (HHO (Heidari et al. 2019) \& EO (Faramarzi et al. 2019)), PSO variants (HEPSO (Mahmoodabadi et al. 2014) \& RPSOLF (Yan et al. 2017)), DE variants (JADE (Zhang and Sanderson 2009) \& SHADE (Tanabe and Fukunaga 2013)) and hybrid variants (FAPSO (Xia et al. 2018), \& PSOSCALF (Chegini et al. 2018)). Table 2 lists the parameters of all of the above-mentioned compared and proposed algorithms. Stopping criteria, independent run and population size of presented algorithms are taken as same or the least of comparative methods for fair comparison. Table 3 shows the comparative experimental results of 30 independent runs in terms of mean/average value (avg), standard deviation (std), and ranking (rank) of the objective function values. The comparative results of the algorithms directly from the original papers. 
Table 1 Classical unconstrained benchmark functions ( $\left.\operatorname{cubf}_{\mathrm{s}}\right)$

\begin{tabular}{|c|c|c|c|c|}
\hline cubf $_{\mathrm{s}}$ formulation & Type & Dim. & Range & $f_{\min }$ \\
\hline$f_{1}(x)=\sum_{i=1}^{D} x_{i}^{2}$ & \multirow{7}{*}{$\begin{array}{l}\text { 馬 } \\
\text { 音 } \\
\text { 严 }\end{array}$} & 30 & {$[-100,100]$} & 0 \\
\hline$f_{2}(x)=\sum_{i=1}^{D}|x|+\prod_{i=1}^{D}\left|x_{i}\right|$ & & 30 & {$[-10,10]$} & 0 \\
\hline$f_{3}(x)=\sum_{i=1}^{D}\left(\sum_{j=1}^{i} x_{j}\right)^{2}$ & & 30 & {$[-100,100]$} & 0 \\
\hline$f_{4}(x)=\max _{i}\left|x_{i}\right|, 1 \leq i \leq D$ & & 30 & {$[-100,100]$} & 0 \\
\hline$f_{5}(x)=\sum_{i}^{D-1}\left[100\left(x_{i+1}-x_{i}^{2}\right)^{2}+\left(x_{i}-1\right)^{2}\right]$ & & 30 & {$[-30,30]$} & 0 \\
\hline$f_{6}(x)=\sum_{i}^{D}\left(\left\lfloor x_{i}+0.5\right\rfloor\right)^{2}$ & & 30 & {$[-100,100]$} & 0 \\
\hline$f_{7}(x)=\left(\sum_{i}^{D} i x_{i}^{4}\right)+\operatorname{rand}[0,1)$ & & 30 & {$[-1.28,1.28]$} & 0 \\
\hline$f_{8}(x)=\sum_{i}^{D}-x_{i} \sin \left(\sqrt{\left|x_{i}\right|}\right)$ & \multirow{6}{*}{ 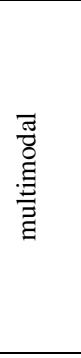 } & 30 & {$[-500,500]$} & $-418.9829 * \mathrm{D}$ \\
\hline$f_{9}(x)=\sum_{i}^{D}\left[x_{i}^{2}-10 \cos (2 \pi x i)+10\right]$ & & 30 & {$[-5.12,5.12]$} & 0 \\
\hline$f_{10}(x)=-20 \exp \left(-\sqrt[0.2]{\frac{1}{D}} \sum_{i}^{D} x_{i}^{2}\right)-\exp \left(\frac{1}{D} \sum_{i}^{D} \cos 2 \pi x i\right)+20+e$ & & 30 & {$[-32,32]$} & 0 \\
\hline$f_{11}(x)=\frac{1}{4000} \sum_{i}^{D} x_{i}^{2}-\prod_{i}^{D} \cos \left(\frac{x_{i}}{\sqrt{i}}\right)+1$ & & 30 & {$[-600,600]$} & 0 \\
\hline $\begin{aligned} f_{12}(x)= & \frac{\pi}{D}\left\{10 \sin ^{2}(x y)+\sum_{i}^{D}\left(x_{i}-1\right)^{2}\left(1+\sin ^{2}\left(x y_{i+1}\right)\right)+\left(y_{D}-1\right)^{2}\right\}+ \\
& \sum_{i}^{D} U\left(x_{i}, 10,100,4\right)\end{aligned}$ & & 30 & {$[-50,50]$} & 0 \\
\hline $\begin{aligned} f_{13}(x)= & 0.1\left\{\sin ^{2}\left(3 \pi x_{i}\right)\right\}+\sum_{i=1}^{D}\left(x_{i}-1\right)^{2}\left[1+\sin ^{2}\left(3 \pi x_{i}+1\right)\right]+\left(x_{D}-1\right)^{2}+ \\
& \sum_{i}^{D} U\left(x_{i}, 5,100,4\right)\end{aligned}$ & & 30 & {$[-50,50]$} & 0 \\
\hline$f_{14}(x)=\left(\frac{1}{500}+\sum_{j=1}^{25}\left(\frac{1}{j+1+\sum_{i=0}^{1}\left(x_{i}-a_{i j}\right)^{6}}\right)\right)^{-1}$ & \multirow{10}{*}{ 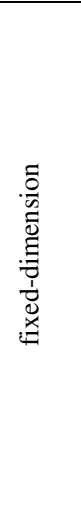 } & 2 & $[-65,65]]$ & 1 \\
\hline$f_{15}(x)=\sum_{i=0}^{10}\left(a_{i}-\frac{x_{0}\left(b_{i}^{2}+b_{i} x_{1}\right)}{\left(b_{i}^{2}+b_{i} x_{2}+x_{3}\right)}\right)^{2}$ & & 4 & {$[-5,5]$} & 0.00030 \\
\hline$f_{16}(x)=4 x_{0}^{2}-2.1 x_{0}^{4}+\frac{1}{3} x_{0}^{6}+x_{0} x_{1}-4 x_{1}^{2}+4 x_{1}^{4}$ & & 2 & {$[-5,5]$} & -1.0316 \\
\hline$f_{17}(x)=\left(x_{1}-\frac{5.1}{4 \pi^{2}} x_{0}^{2}+\frac{5}{\pi} x_{0}-6\right)^{2}+10\left(1-\frac{1}{8 \pi}\right) \cos x_{0}+10$ & & 2 & {$[-5,5]$} & 0.398 \\
\hline $\begin{aligned} f_{18}(x)= & 1+\left(x_{0}+x_{1}+1\right)^{2}\left(19-14 x_{0}+3 x_{0}^{2}-14 x_{1}-6 x_{0} x_{1}+3 x_{1}^{2}\right)\{30+ \\
& \left.\left(2 x_{0}-3 x_{1}\right)^{2}\left(18-32 x_{0}+12 x_{0}^{2}+48 x_{1}-36 x_{0} x_{1}+27 x_{1}^{2}\right)\right\}\end{aligned}$ & & 2 & {$[-2,2]$} & 3 \\
\hline$f_{19}(x)=-\sum_{i=1}^{4} c_{i} \exp \left(-\sum_{j=1}^{3} a_{i j}\left(x_{j}-p_{i j}\right)^{2}\right)$ & & 3 & {$[1,3]$} & -3.86 \\
\hline$f_{20}(x)=-\sum_{i=1}^{4} c_{i} \exp \left(-\sum_{j=1}^{6} a_{i j}\left(x_{j}-p_{i j}\right)^{2}\right)$ & & 6 & {$[0,1]$} & -3.32 \\
\hline$f_{21}(x)=-\sum_{i=1}^{5}\left(\left(x-a_{i}\right)^{T}\left(x-a_{i}\right)+c_{i}\right)^{-1}$ & & 4 & {$[0,10]$} & -10.1532 \\
\hline$f_{22}(x)=-\sum_{i=1}^{7}\left(\left(x-a_{i}\right)^{T}\left(x-a_{i}\right)+c_{i}\right)^{-1}$ & & 4 & {$[0,10]$} & -10.4028 \\
\hline$f_{23}(x)=-\sum_{i=1}^{10}\left(\left(x-a_{i}\right)^{T}\left(x-a_{i}\right)+c_{i}\right)^{-1}$ & & 4 & {$[0,10]$} & -10.5363 \\
\hline
\end{tabular}

Table 2 Parameter for cubf $_{\mathrm{s}}$ of compared and presented algorithms

\begin{tabular}{|c|c|c|c|c|c|c|}
\hline \multirow{2}{*}{ Algorithm } & \multirow{2}{*}{ Reference } & \multicolumn{2}{|l|}{ Control Parameter } & \multirow{2}{*}{$\begin{array}{l}\text { Population } \\
\text { Size } \\
\end{array}$} & \multirow[t]{2}{*}{ Stopping criterion } & \multirow[t]{2}{*}{ Run } \\
\hline & & Term & Values & & & \\
\hline $\mathrm{HHO}$ & (Heidari et al. 2019) & escaping energy & $E<0.5, E \geq 0.5$ & 30 & 500 & 30 \\
\hline EO & (Faramarzi et al. 2019) & $a_{1}, a_{2}, \mathrm{GP}$ & $\{1,1.5,2,2.5,3\},\{0.1,0.5,1,1.5,2\},(0.1 .0 .25,0.5,0.75,0.9\}$ & 30 & 500 & 30 \\
\hline HEPSO & (Mahmoodabadi et al. 2014) & $\mathrm{P}_{\mathrm{C}}, \mathrm{P}_{\mathrm{B}}$ & $0.95,0.02$ & 50 & 500 & 30 \\
\hline RPSOLF & (Yan et al. 2017) & $w, \mathrm{c}_{1}, \mathrm{c}_{2}, \mathrm{c}_{3}, \beta, \varepsilon$ & $0.55,1.49,1.49,1.5,0.99$ & 50 & 500 & 30 \\
\hline JADE & (Zhang and Sanderson 2009) & $\mathrm{F}_{i}, C R_{i}$ & $\operatorname{randc}_{\mathrm{i}}\left(\mu_{\mathrm{F}}, 0.1\right), \operatorname{randn}_{\mathrm{i}}\left(\mu_{C R}, 0.1\right)$ & 50 & 1000 & 30 \\
\hline SHADE & (Tanabe and Fukunaga 2013) & Pbest, Arc rate & $0.1,2$ & 30 & 500 & 30 \\
\hline FAPSO & (Xia et al. 2018) & - & - & 50 & 5000 & 30 \\
\hline PSOSCALF & (Chegini et al. 2018) & $w_{\min }, w_{\max }, \mathrm{c}_{1 \min }, \mathrm{c}_{1 \max }, \mathrm{c}_{2 \min }, \mathrm{c}_{2 \max }, \beta$ & $0.4,0.9,0.5,2.5,0.5,2.5,1.5$ & 50 & 500 & 30 \\
\hline nPSO & $\widetilde{\Xi} \Xi$ & - & - & 30 & 500 & 30 \\
\hline $\mathrm{nDE}$ & 总吾 & $\tau$ & {$[1.5,2.2]$} & 30 & 500 & 30 \\
\hline i $h$ PSODE & 党 & - & - & 30 & 500 & 30 \\
\hline
\end{tabular}

It should have been noted that the average objective function values of the presented algorithms (nPSO, $\mathrm{nDE}$ and ihPSODE) are better and/or equal to the compared standard algorithms, PSO alternatives, DE alternatives, and hybrid variants, as shown in Table 3. The presented algorithms produce less std for most of the cubfs which terms their stability. Also, all algorithms are ranked separately (as 1 for best, 2 for following performer and so on) grounded on average result values in Table 3. From Table 3 it is decided that $i h P S O D E, n D E \&$ nPSO ranked as $1^{\text {st }}, 2^{\text {nd }} \& 4^{\text {th }}$ successively. As well, average and overall rank of presented versus others algorithms are declared in Table 3 . Then, it can be say that the proposed algorithms outperform others by rankings. Furthermore, the supremacy of the proposed algorithms over other algorithms is analyzed statistically using- (i) a one-tailed t-test (at $5 \%$ significance level of 98 degrees of freedom (df)) and (ii) Wilcoxon Signed Rank (WSR) test (at 5\% significance level). The details of these tests can be found in (Das and Parouha 2015). In Table 4, results

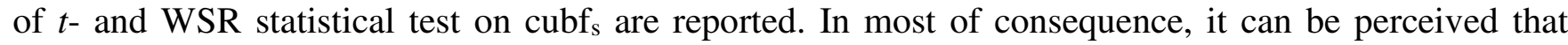
from this table presented algorithms has ' $\mathrm{a}^{+}$' or ' $\mathrm{a}$ ' sign in case of t-test which signify highly or significantly better than other respectively and ' + ' or ' $\approx$ ' sign in case WSR test which indicate better or equally performances. Moreover, $p$-values of the presented algorithms listed in Table 4 which is lower than others and indicating that their reliability to produce best results on the majority of runs. 
Table 3 Simulation results on cubf $\mathrm{s}_{\mathrm{s}}$

\begin{tabular}{|c|c|c|c|c|c|c|c|c|c|c|c|c|}
\hline \multirow[t]{3}{*}{$f_{n}(x)$} & \multirow{3}{*}{ Criteria } & \multicolumn{11}{|c|}{ Algorithms } \\
\hline & & \multicolumn{2}{|c|}{ Classical algorithms } & \multicolumn{2}{|c|}{ PSO alternatives } & DE alte & atives & Hybrid & ernatives & & sented algoritl & \\
\hline & & $\mathrm{HHO}$ & EO & HEPSO & RPSOLF & JADE & SHADE & FAPSO & PSOSCALF & nPSO & $\mathrm{nDE}$ & i $h$ PSODE \\
\hline$f_{1}(x)$ & avg & $2.03 \mathrm{e}+00$ & $3.32 \mathrm{e}-40$ & 16.26772 & $5.065 \mathrm{e}-269$ & $1.87 \mathrm{e}-31$ & $1.42 \mathrm{e}-09$ & $2.87 \mathrm{e}-127$ & $1.11014 \mathrm{e}-20$ & $\mathbf{0}$ & $\mathbf{0}$ & $\mathbf{0}$ \\
\hline & std & $4.04 \mathrm{e}-01$ & $6.78 \mathrm{e}-40$ & 10.01293 & $0.00 e+00$ & $6.43 e-31$ & $3.09 \mathrm{e}-09$ & $1.76 \mathrm{e}-127$ & $1.83289 \mathrm{e}-20$ & $\mathbf{0}$ & $\mathbf{0}$ & $\mathbf{0}$ \\
\hline & rank & 9 & 4 & 8 & 2 & 5 & 7 & 3 & 6 & 1 & 1 & 1 \\
\hline$f_{2}(x)$ & avg & $1.70 \mathrm{e}+00$ & $7.12 \mathrm{e}-23$ & 1.28424 & $1.000 \mathrm{e}-134$ & $2.79 \mathrm{e}-15$ & 0.0087 & $1.02 \mathrm{e}-17$ & $4.09460 \mathrm{e}-11$ & $\mathbf{0}$ & $\mathbf{0}$ & $\mathbf{0}$ \\
\hline & std & $7.37 \mathrm{e}-02$ & $6.36 \mathrm{e}-23$ & 0.41611 & $3.753 \mathrm{e}-134$ & $9.51 \mathrm{e}-15$ & 0.0213 & $1.43 \mathrm{e}-17$ & $5.68981 \mathrm{e}-11$ & $\mathbf{0}$ & $\mathbf{0}$ & $\mathbf{0}$ \\
\hline & rank & 9 & 3 & 8 & 2 & 4 & 7 & 5 & 6 & 1 & 1 & 1 \\
\hline$f_{3}(x)$ & avg & $1.17 \mathrm{e}+02$ & $8.06 \mathrm{e}-09$ & $7.423 e+03$ & $7.791 \mathrm{e}-249$ & $1.10 \mathrm{e}-03$ & 15.4352 & $1.68 \mathrm{e}-11$ & $2.16858 \mathrm{e}-12$ & $0.17 \mathrm{e}-129$ & $\mathbf{0}$ & $\mathbf{0}$ \\
\hline & std & $5.28 \mathrm{e}+00$ & $1.60 \mathrm{e}-08$ & $7.423 e+03$ & $0.00 \mathrm{e}+00$ & $5.14 \mathrm{e}-03$ & 9.9489 & $2.49 \mathrm{e}-11$ & $1.03815 \mathrm{e}-11$ & $1.67 \mathrm{e}-131$ & 0 & $\mathbf{0}$ \\
\hline & rank & 9 & 6 & 10 & 2 & 7 & 8 & 5 & 4 & 3 & 1 & 1 \\
\hline$f_{4}(x)$ & avg & $2.05 \mathrm{e}+00$ & $5.39 \mathrm{e}-10$ & 23.95145 & $1.937 \mathrm{e}-157$ & $1.66 \mathrm{e}-03$ & 0.9796 & $4.09 e+03$ & $8.47410 \mathrm{e}-08$ & $8.35 \mathrm{e}-098$ & $3.28 \mathrm{e}-101$ & 0 \\
\hline & std & $7.40 \mathrm{e}-02$ & $1.38 \mathrm{e}-09$ & 7.71460 & $1.061 \mathrm{e}-156$ & $1.98 \mathrm{e}-03$ & 0.7995 & $6.53 \mathrm{e}+02$ & $1.23324 \mathrm{e}-07$ & $7.08 \mathrm{e}-098$ & $7.12 \mathrm{e}-103$ & 0 \\
\hline & rank & 9 & 5 & 10 & 2 & 7 & 8 & 11 & 6 & 4 & 3 & 1 \\
\hline$f_{5}(x)$ & avg & $2.95 \mathrm{e}+00$ & $2.53 \mathrm{e}+01$ & $2.380 \mathrm{e}+03$ & 27.42672 & $1.18 \mathrm{e}+01$ & 24.4743 & $6.55 \mathrm{e}-11$ & 21.97646 & $4.85 \mathrm{e}-012$ & $1.25 \mathrm{e}-021$ & $2.21 \mathrm{e}-033$ \\
\hline & std & $8.36 \mathrm{e}-02$ & $0.16 \mathrm{e}+00$ & $1.852 \mathrm{e}+03$ & 0.24848 & $1.57 \mathrm{e}+01$ & 11.2080 & $1.99 \mathrm{e}-11$ & 0.54774 & $3.21 \mathrm{e}-012$ & $1.85 \mathrm{e}-023$ & $3.72 \mathrm{e}-037$ \\
\hline & rank & 9 & 7 & 11 & 8 & 10 & 6 & 4 & 5 & 3 & 2 & 1 \\
\hline$f_{6}(x)$ & avg & $2.49 \mathrm{e}+00$ & $8.29 \mathrm{e}-06$ & 21.55405 & 2.98244 & $4.59 \mathrm{e}-31$ & $5.31 \mathrm{e}-10$ & $2.37 \mathrm{e}-12$ & $7.13998 \mathrm{e}-12$ & $1.75 e-032$ & 0 & 0 \\
\hline & std & $8.25 \mathrm{e}-02$ & $5.02 \mathrm{e}-06$ & 9.33263 & 0.23250 & $1.65 \mathrm{e}-30$ & $6.35 \mathrm{e}-10$ & $1.84 \mathrm{e}-13$ & $3.65884 \mathrm{e}-11$ & $9.16 \mathrm{e}-035$ & 0 & 0 \\
\hline & rank & 9 & 8 & 6 & 10 & 3 & 7 & 4 & 5 & 2 & 1 & 1 \\
\hline$f_{7}(x)$ & avg & $8.20 \mathrm{e}+00$ & $1.17 \mathrm{e}-02$ & 0.12982 & 0.00104 & $6.49 \mathrm{e}-03$ & 0.0235 & 0 & 0.00012 & $5.11 \mathrm{e}-001$ & $2.19 \mathrm{e}-003$ & $1.07 \mathrm{e}-003$ \\
\hline & std & $1.69 \mathrm{e}-01$ & $6.54 \mathrm{e}-04$ & 0.09727 & $7.644 \mathrm{e}-04$ & $2.48 \mathrm{e}-03$ & 0.0088 & 0 & 0.00010 & $1.70 \mathrm{e}-002$ & $1.10 \mathrm{e}-004$ & $1.40 \mathrm{e}-005$ \\
\hline & rank & 11 & 6 & 10 & 7 & 5 & 9 & 1 & 4 & 8 & 3 & 2 \\
\hline$f_{8}(x)$ & avg & $4.86 \mathrm{e}+00$ & -9016.34 & $-2.139 e+03$ & $-3.254 e+03$ & $-1.24 e+04$ & -11713.1 & $2.48 \mathrm{e}-11$ & -12569.48 & $-6.37 e+004$ & $-1.25 e+004$ & $-1.25 e+004$ \\
\hline & std & $1.03 \mathrm{e}+00$ & 595.1113 & $8.282 \mathrm{e}+02$ & $2.860 \mathrm{e}+02$ & $1.27 \mathrm{e}+02$ & 230.49 & $6.44 \mathrm{e}-12$ & $2.39996 \mathrm{e}-07$ & $2.10 \mathrm{e}-001$ & $1.07 \mathrm{e}-017$ & 0 \\
\hline & rank & 4 & 6 & 5 & 7 & 1 & 8 & 2 & 1 & 9 & 1 & 1 \\
\hline$f_{9}(x)$ & avg & $3.77 \mathrm{e}+00$ & 0 & 42.00118 & 0 & $1.71 \mathrm{e}-04$ & 8.5332 & 0 & 0 & 0 & 0 & 0 \\
\hline & std & $8.87 \mathrm{e}-01$ & 0 & 7.08632 & 0 & $1.52 \mathrm{e}-04$ & 2.1959 & 0 & 0 & 0 & 0 & 0 \\
\hline & rank & 3 & 1 & 5 & 1 & 2 & 4 & 1 & 1 & 1 & 1 & 1 \\
\hline$f_{10}(x)$ & avg & $3.75 e+00$ & $8.34 \mathrm{e}-14$ & 2.83842 & $4.085 \mathrm{e}-15$ & $1.31 \mathrm{e}-14$ & 0.3957 & $4.86 \mathrm{e}-15$ & $2.24609 \mathrm{e}-11$ & $1.97 \mathrm{e}-014$ & $1.44 \mathrm{e}-015$ & $2.88 \mathrm{e}-016$ \\
\hline & std & $8.75 e-01$ & $2.53 \mathrm{e}-14$ & 0.66134 & $1.084 \mathrm{e}-15$ & $2.46 \mathrm{e}-14$ & 0.5868 & $1.74 \mathrm{e}-15$ & $2.33542 \mathrm{e}-11$ & 0 & 0 & 0 \\
\hline & rank & 8 & 7 & 9 & 3 & 5 & 10 & 4 & 8 & 6 & 2 & 1 \\
\hline$f_{11}(x)$ & avg & $4.17 \mathrm{e}+00$ & 0 & 1.16858 & 0 & $2.87 \mathrm{e}-03$ & 0.0048 & $1.74 \mathrm{e}-16$ & 0 & $3.37 \mathrm{e}-111$ & 0 & 0 \\
\hline & std & $5.56 \mathrm{e}-01$ & 0 & 0.12602 & 0 & $7.85 \mathrm{e}-03$ & 0.0077 & $3.60 \mathrm{e}-16$ & 0 & $1.11 \mathrm{e}-119$ & 0 & 0 \\
\hline & rank & 7 & 1 & 6 & 1 & 4 & 5 & 3 & 1 & 2 & 1 & 1 \\
\hline$f_{12}(x)$ & avg & $1.90 \mathrm{e}+01$ & 7.97e-07 & 0.47856 & 0.26157 & $1.73 \mathrm{e}-02$ & 0.0346 & $1.57 \mathrm{e}-32$ & $8.46465 \mathrm{e}-14$ & $3.34 \mathrm{e}-002$ & $1.05 \mathrm{e}-032$ & $3.73 \mathrm{e}-033$ \\
\hline & std & $3.31 \mathrm{e}+00$ & $7.69 \mathrm{e}-07$ & 0.22623 & 0.03386 & $7.74 \mathrm{e}-02$ & 0.0875 & $0.00 \mathrm{e}+00$ & $2.79106 \mathrm{e}-13$ & $1.02 \mathrm{e}-004$ & $2.77 \mathrm{e}-034$ & $3.18 \mathrm{e}-034$ \\
\hline & rank & 11 & 5 & 10 & 9 & 6 & 8 & 3 & 4 & 7 & 2 & 1 \\
\hline$f_{13}(x)$ & avg & $1.89 \mathrm{e}+01$ & 0.029295 & 1.85056 & 2.05282 & $5.45 \mathrm{e}-24$ & $7.32 \mathrm{e}-04$ & $1.58 \mathrm{e}-32$ & 0.00399 & $9.30 \mathrm{e}-004$ & $2.09 \mathrm{e}-021$ & $1.58 \mathrm{e}-032$ \\
\hline & std & $1.56 \mathrm{e}+00$ & 0.035271 & 0.65246 & 0.16579 & $2.58 \mathrm{e}-23$ & 0.0028 & 0 & 0.00928 & $3.71 \mathrm{e}-004$ & $3.27 \mathrm{e}-023$ & $1.89 \mathrm{e}-043$ \\
\hline & rank & 10 & 7 & 8 & 9 & 2 & 4 & 0 & 6 & 5 & 3 & 1 \\
\hline$f_{14}(x)$ & avg & $9.98 \mathrm{e}-01$ & 0.99800 & 0.99800 & 1.54064 & $9.98 \mathrm{e}-01$ & 0.998004 & $9.98 \mathrm{e}-001$ & 1.13027 & $9.98 \mathrm{e}-001$ & $9.98 \mathrm{e}-001$ & $9.98 \mathrm{e}-001$ \\
\hline & std & $9.23 \mathrm{e}-01$ & $1.54 \mathrm{e}-16$ & $9.219 \mathrm{e}-17$ & 1.84429 & 0 & $5.83 \mathrm{e}-17$ & $1.27 \mathrm{e}-08$ & 0.50338 & 0 & 0 & 0 \\
\hline & rank & 1 & 1 & 1 & 3 & 0 & 1 & 1 & 2 & 0 & 0 & 0 \\
\hline$f_{15}(x)$ & avg & $3.10 \mathrm{e}-04$ & 0.00239 & $6.404 \mathrm{e}-04$ & 0.00171 & $3.01 \mathrm{e}-03$ & 0.002374 & $3.95 \mathrm{e}-04$ & $3.13244 \mathrm{e}-04$ & $3.99 \mathrm{e}-004$ & $3.83 \mathrm{e}-004$ & $3.02 \mathrm{e}-004$ \\
\hline & std & $1.97 \mathrm{e}-04$ & 0.00609 & $2.801 \mathrm{e}-04$ & 0.00508 & $6.92 \mathrm{e}-03$ & 0.0061 & $6.02 \mathrm{e}-08$ & $2.17489 \mathrm{e}-05$ & $2.96 \mathrm{e}-007$ & $9.23 \mathrm{e}-012$ & $2.38 \mathrm{e}-018$ \\
\hline & rank & 2 & 11 & 7 & 9 & 8 & 10 & 5 & 3 & 6 & 4 & 1 \\
\hline$f_{16}(x)$ & avg & $-1.03 e+00$ & -1.03161 & -1.03161 & -1.03161 & $-1.03 e+00$ & -1.03162 & $-1.03 e+00$ & -1.0316 & $-1.03 e+000$ & $-1.03 e+000$ & $-1.03 e+000$ \\
\hline & std & $6.78 \mathrm{e}-16$ & $6.04 \mathrm{e}-16$ & $3.554 \mathrm{e}-15$ & $1.650 \mathrm{e}-05$ & $6.78 \mathrm{e}-16$ & $6.51 \mathrm{e}-16$ & 0 & $4.40244 \mathrm{e}-16$ & 0 & 0 & 0 \\
\hline & rank & 1 & 1 & 1 & 1 & 1 & 1 & 0 & 1 & 0 & 1 & 1 \\
\hline$f_{17}(x)$ & avg & $3.98 \mathrm{e}-01$ & 0.397887 & 0.39787 & 0.39837 & 3.98e- 01 & 0.397887 & $3.98 \mathrm{e}-001$ & 0.39788 & 3.98e-001 & 3.98e-001 & $3.98 \mathrm{e}-001$ \\
\hline & std & $2.54 \mathrm{e}-06$ & 0 & $6.594 \mathrm{e}-13$ & $5.267 \mathrm{e}-04$ & $0.00 \mathrm{e}+00$ & $3.24 \mathrm{e}-16$ & $0.00 \mathrm{e}+000$ & $3.66527 \mathrm{e}-15$ & 0 & 0 & 0 \\
\hline & rank & 1 & 2 & 2 & 1 & 1 & 2 & 1 & 2 & 1 & 1 & 1 \\
\hline$f_{18}(x)$ & avg & 3 & 3 & 0.65246 & 3.00002 & 3 & 3 & 3 & 3 & 3 & 3 & 3 \\
\hline & std & 0 & $1.56 \mathrm{e}-15$ & $5.146 \mathrm{e}-11$ & $1.658 \mathrm{e}-05$ & $1.82 \mathrm{e}-15$ & $1.87 \mathrm{e}-15$ & $5.31 \mathrm{e}-016$ & $5.96540 \mathrm{e}-13$ & $1.33 \mathrm{e}-018$ & 0 & 0 \\
\hline & rank & 1 & 1 & 2 & 1 & 1 & 1 & 1 & 1 & 1 & 1 & 1 \\
\hline$f_{19}(x)$ & avg & $-3.86 e+00$ & -3.86278 & -3.86278 & -3.85923 & $-3.86 e+00$ & -3.86278 & $-3.87 e+000$ & -3.86278 & $-3.86 \mathrm{e}+000$ & $-3.86 \mathbf{e}+000$ & $-3.86 e+000$ \\
\hline & std & $2.44 \mathrm{e}-03$ & $2.59 \mathrm{e}-15$ & $1.008 \mathrm{e}-13$ & 0.00283 & $2.71 \mathrm{e}-15$ & $2.69 \mathrm{e}-15$ & $2.11 \mathrm{e}-004$ & $8.31755 \mathrm{e}-15$ & $3.36 \mathrm{e}-021$ & 0 & 0 \\
\hline & rank & 1 & 1 & 1 & 3 & 1 & 1 & 2 & 1 & 1 & 1 & 1 \\
\hline$f_{20}(x)$ & avg & -3.322 & -3.2687 & -3.31803 & -3.10441 & $-3.29 e+00$ & -3.27047 & $-3.29 \mathrm{e}+000$ & -3.27168 & $-3.27 e+000$ & $-3.32 \mathrm{e}+000$ & $-3.32 \mathrm{e}+000$ \\
\hline & std & 0.137406 & 0.05701 & 0.02170 & 0.15760 & $5.11 \mathrm{e}-02$ & 0.0599 & 0 & 0.06371 & 0 & 0 & 0 \\
\hline & rank & 1 & 6 & 2 & 7 & 4 & 5 & 3 & 5 & 5 & 1 & 1 \\
\hline$f_{21}(x)$ & avg & -10.1531 & -8.55481 & -10.15319 & -4.76171 & $-9.14 e+00$ & -9.2343 & $-7.51 \mathrm{e}+00$ & -10.15319 & $-9.87 e+001$ & $-1.01 \mathrm{e}+001$ & $-1.01 \mathrm{e}+001$ \\
\hline & std & 0.885673 & 2.76377 & $2.680 \mathrm{e}-05$ & 0.73723 & $2.06 \mathrm{e}+00$ & 1.3969 & $1.21 \mathrm{e}-01$ & $4.46227 \mathrm{e}-15$ & $1.07 \mathrm{e}-011$ & 0 & 0 \\
\hline & rank & 1 & 5 & 1 & 6 & 4 & 3 & 3 & 1 & 2 & 1 & 1 \\
\hline$f_{22}(x)$ & avg & -10.4015 & -9.3353 & -10.39978 & -4.81927 & $-9.88 \mathrm{e}+00$ & -10.2809 & $-6.04 e+01$ & -10.40294 & $-9.87 e+000$ & $-1.04 e+001$ & $-1.04 \mathrm{e}+001$ \\
\hline & std & 1.352375 & 2.43834 & 0.01728 & 0.75699 & $1.61 \mathrm{e}+00$ & 1.3995 & $2.14 \mathrm{e}-01$ & $1.80672 \mathrm{e}-15$ & $2.08 \mathrm{e}-002$ & $2.91 \mathrm{e}-012$ & $1.10 \mathrm{e}-016$ \\
\hline & rank & 1 & 5 & 1 & 7 & 3 & 2 & 6 & 1 & 4 & 1 & 1 \\
\hline$f_{23}(x)$ & avg & -10.5364 & -9.63655 & -10.53640 & -5.06376 & $-1.03 e+01$ & 63.333 & $-5.64 \mathrm{e}+00$ & -10.53640 & $-9.13 e+000$ & $-1.05 e+001$ & $-1.05 e+001$ \\
\hline & std & 0.927655 & 2.38811 & $5.845 \mathrm{e}-07$ & 0.82968 & $1.40 \mathrm{e}+00$ & 80.872 & $5.70 \mathrm{e}-02$ & $4.84794 \mathrm{e}-15$ & $2.05 \mathrm{e}-003$ & $1.07 \mathrm{e}-021$ & 0 \\
\hline & rank & 1 & 3 & 1 & 6 & 2 & 7 & 5 & 1 & 4 & 1 & 1 \\
\hline Sumo & & 119 & 102 & 125 & 107 & 87 & 124 & 75 & 75 & 78 & 35 & 24 \\
\hline & & 5.17 & 4.43 & 5.43 & 4.65 & 3.78 & 5.39 & 3.26 & 3.26 & 3.39 & 1.52 & 1.04 \\
\hline Overall & & 8 & 6 & 10 & 7 & 5 & 9 & 3 & 3 & 4 & 2 & 1 \\
\hline
\end{tabular}

Table 4 Statistical outcomes on cubf $_{\mathrm{s}}$

\begin{tabular}{|c|c|c|c|c|c|c|c|c|c|c|c|}
\hline \multirow{3}{*}{ Vs } & \multirow{3}{*}{ Standards } & \multicolumn{10}{|c|}{ Algorithm } \\
\hline & & \multicolumn{2}{|c|}{ Classical algorithms } & \multicolumn{2}{|c|}{ PSO alternatives } & \multicolumn{2}{|c|}{ DE alternatives } & \multicolumn{2}{|c|}{ Hybrid alternatives } & \multicolumn{2}{|c|}{ Presented algorithms } \\
\hline & & $\mathrm{HHO}$ & EO & HEPSO & RPSOLF & JADE & SHADE & FAPSO & PSOSCALF & $\mathrm{nDE}$ & ihPSODE \\
\hline \multirow{8}{*}{$\begin{array}{l}0 \\
\infty \\
\text { â }\end{array}$} & Better & 11 & 21 & 20 & 19 & 20 & 13 & 15 & 19 & 0 & 0 \\
\hline & Equal & 4 & 2 & 2 & 2 & 2 & 9 & 4 & 3 & 7 & 8 \\
\hline & Worst & 8 & 0 & 1 & 2 & 1 & 1 & 4 & 1 & 16 & 15 \\
\hline & $\mathrm{R}^{+}$ & 293 & 387 & 312 & 323 & 335 & 305 & 300 & 382 & 350 & 400 \\
\hline & $\mathrm{R}^{-}$ & 172 & 78 & 153 & 142 & 130 & 160 & 165 & 83 & 115 & 65 \\
\hline & $p$-value & $5.1 \mathrm{e}-09$ & $5.3 \mathrm{e}-10$ & $5.7 \mathrm{e}-10$ & $5.1 \mathrm{e}-09$ & $6.2 \mathrm{e}-10$ & $4.6 \mathrm{e}-08$ & $5.8 \mathrm{e}-10$ & $5.6 \mathrm{e}-10$ & $6.2 \mathrm{e}-09$ & $5.3 \mathrm{e}-10$ \\
\hline & $t$-test & $\mathrm{a}$ & a & a & $a+$ & a & at & $a+$ & a+ & a & a+ \\
\hline & Decision & $\approx$ & $\approx$ & $\approx$ & + & + & + & + & + & + & + \\
\hline \multirow[t]{4}{*}{ Vs } & & HHO & EO & HEPSO & RPSOLF & JADE & SHADE & FAPSO & PSOSCALF & nPSO & ihPSODE \\
\hline & Better & 15 & 21 & 21 & 21 & 20 & 13 & 14 & 18 & 15 & 0 \\
\hline & Equal & 8 & 2 & 2 & 2 & 3 & 9 & 9 & 5 & 8 & 16 \\
\hline & Worst & 0 & 0 & 0 & 0 & 0 & 1 & 0 & 0 & 0 & 7 \\
\hline \multirow{5}{*}{ 㖗 } & $\mathrm{R}^{+}$ & 416 & 313 & 329 & 465 & 345 & 355 & 377 & 323 & 342 & 315 \\
\hline & $\mathrm{R}^{-}$ & 49 & 152 & 136 & 79 & 120 & 130 & 88 & 142 & 123 & 150 \\
\hline & $p$-value & $5.6 \mathrm{e}-10$ & $5.2 \mathrm{e}-10$ & $6.2 \mathrm{e}-10$ & $6.9 \mathrm{e}-07$ & $8.2 \mathrm{e}-10$ & $5.8 \mathrm{e}-10$ & $6.2 \mathrm{e}-11$ & $4.3 \mathrm{e}-09$ & $5.1 \mathrm{e}-10$ & $6.9 \mathrm{e}-07$ \\
\hline & $t$-test & $\mathrm{a}$ & a & a & $\mathrm{a}$ & $\mathrm{a}$ & at & $a+$ & $\mathrm{a}$ & $a+$ & a+ \\
\hline & Decision & + & + & + & $\approx$ & + & $\approx$ & $\approx$ & + & + & + \\
\hline \multirow[t]{3}{*}{ Vs } & & HHO & EO & HEPSO & RPSOLF & JADE & SHADE & FAPSO & PSOSCALF & $\mathrm{nDE}$ & nPSO \\
\hline & Better & 14 & 0 & 20 & 20 & 20 & 13 & 14 & 15 & 8 & 15 \\
\hline & Equal & 7 & 6 & 3 & 3 & 3 & 10 & 9 & 8 & 15 & 8 \\
\hline \multirow{6}{*}{ 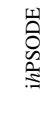 } & Worst & 2 & 17 & 0 & 0 & 0 & 0 & 0 & 0 & 0 & 0 \\
\hline & $\mathrm{R}^{+}$ & 321 & 294 & 330 & 313 & 329 & 367 & 293 & 377 & 323 & 304 \\
\hline & $\mathrm{R}^{-}$ & 144 & 171 & 135 & 152 & 136 & & 172 & 88 & 142 & \\
\hline & $p$-value & $6.2 \mathrm{e}-10$ & $5.1 \mathrm{e}-10$ & $5.1 \mathrm{e}-07$ & $5.1 \mathrm{e}-10$ & $4.6 \mathrm{e}-08$ & $5.7 \mathrm{e}-10$ & $6.2 \mathrm{e}-09$ & $5.3 \mathrm{e}-08$ & 4.6e-10 & $5.7 \mathrm{e}-07$ \\
\hline & $t$-test & $\mathrm{a}$ & $\mathrm{a}$ & a & $a+$ & $\mathrm{a}$ & $a+$ & $\mathrm{a}$ & a+ & $a+$ & $\mathrm{a}$ \\
\hline & Decision & + & + & + & $\approx$ & $\approx$ & + & $\approx$ & + & + & + \\
\hline
\end{tabular}


To demonstrate that presented algorithms satisfied convergence speed, the convergence curves of comparative and presented algorithms on eight $\left(f_{1}(x), f_{5}(x), f_{6}(x), f_{7}(x), f_{8}(x), f_{9}(x), f_{10}(x)\right.$ and $f_{11}(x)$ ) typical 30-D cubf s $_{\mathrm{s}}$ are plotted and presented separately in Fig. 4(a-h). From this figure it can be see that almost all of the considered benchmark functions, either unimodal or multimodal, would be quickly optimized by the presented algorithms (nPSO, nDE, and i $h$ PSODE).

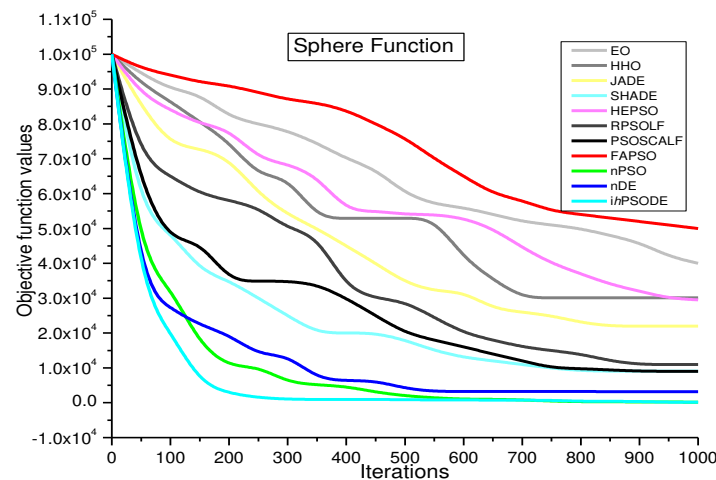

(a). $f_{1}(x)$

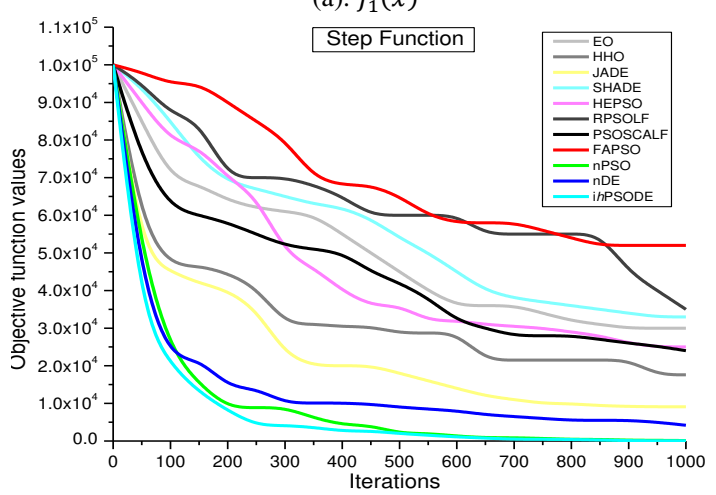

(c). $f_{6}(x)$

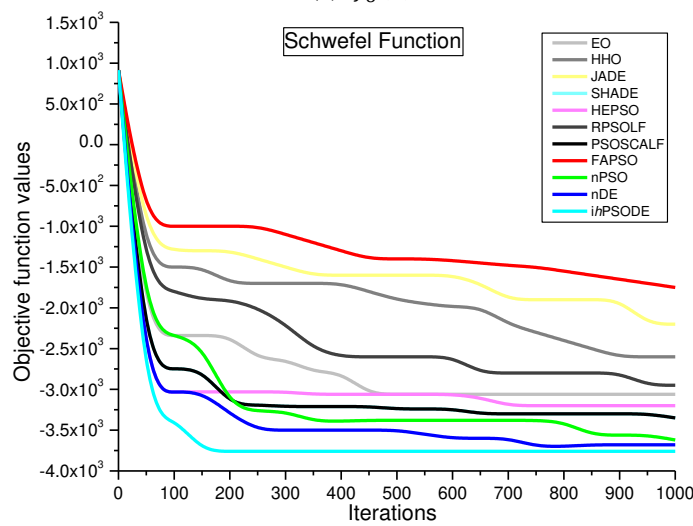

(e). $f_{8}(x)$

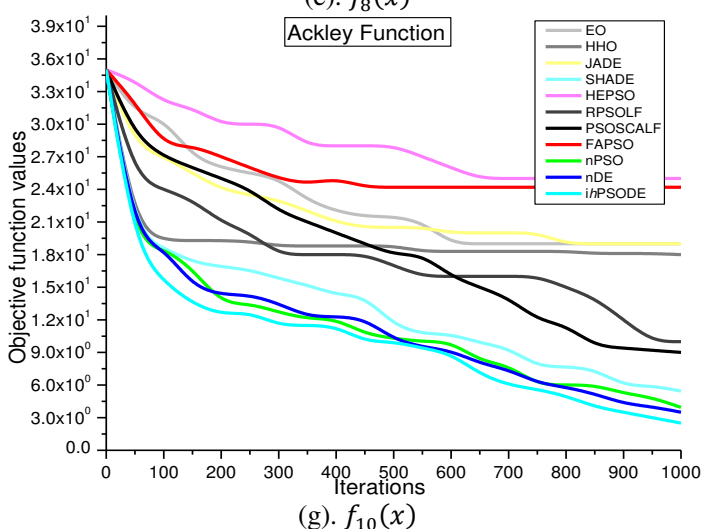

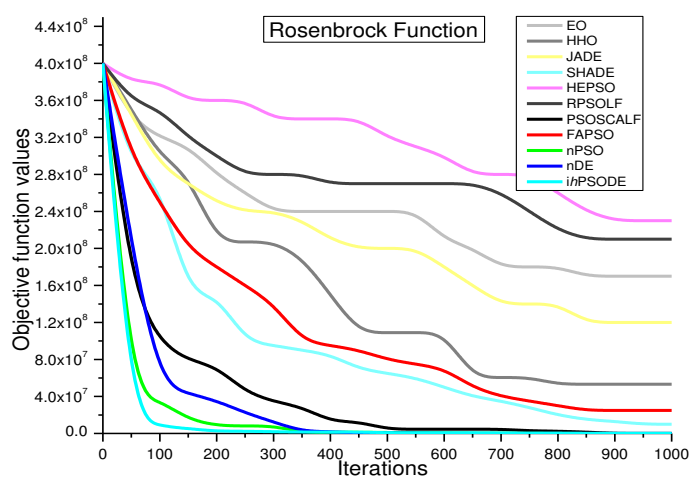

(b). $f_{5}(x)$

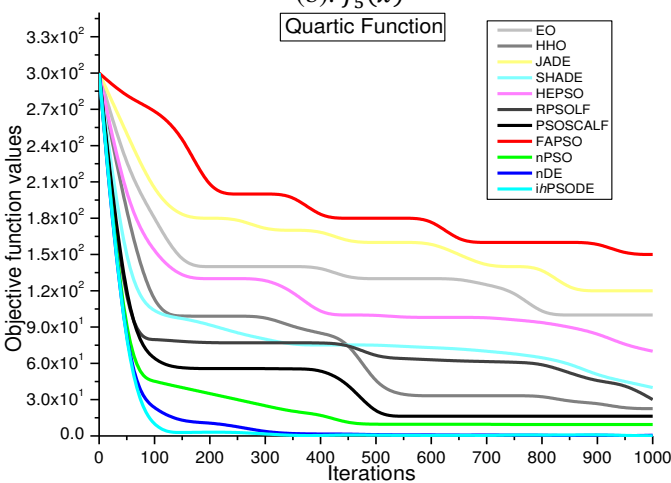

(d). $f_{7}(x)$

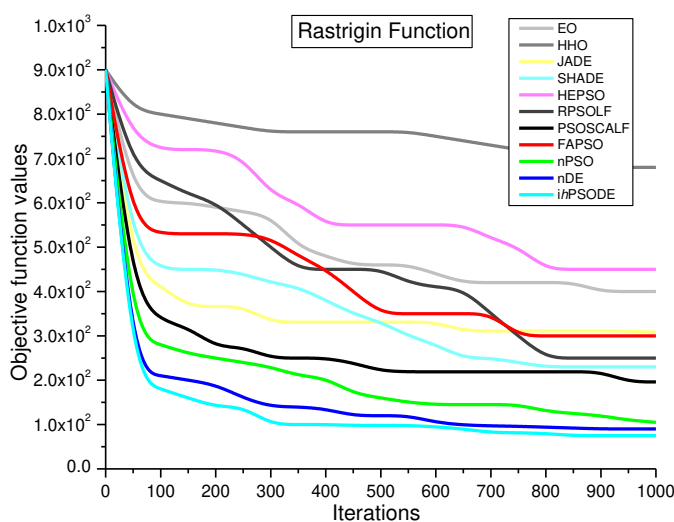

(f). $f_{9}(x)$

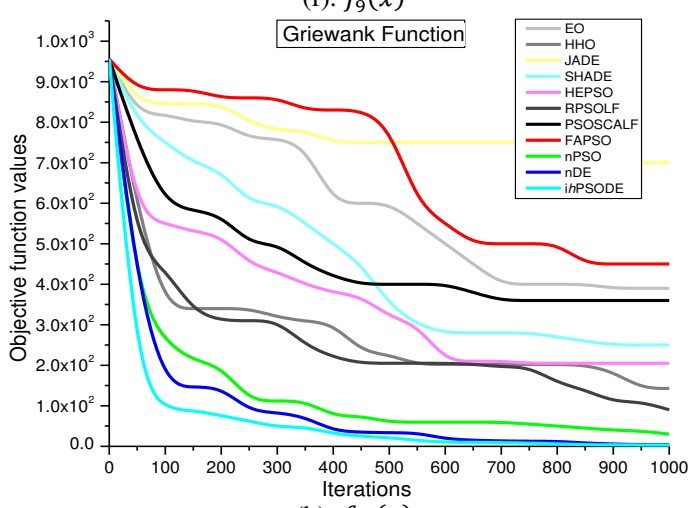

(h). $f_{11}(x)$

Fig. 4(a-h) Convergence of different algorithms 
Likewise, an effort is completed to catch global optimal solution entire of 690 runs (30 population size with 30 runs for each cubf s $_{\text {) }}$ and demonstrated in Fig. 5. It states that the presented algorithms provide the best optimal solutions. Apart from that, the computational time of the presented and equated algorithms on each cubf $\mathrm{s}_{\mathrm{s}}$ is calculated and illustrated in Fig. 6 via box plots. This figure shows that the presented algorithms take less time to attain the best value for the entire set of cubf.

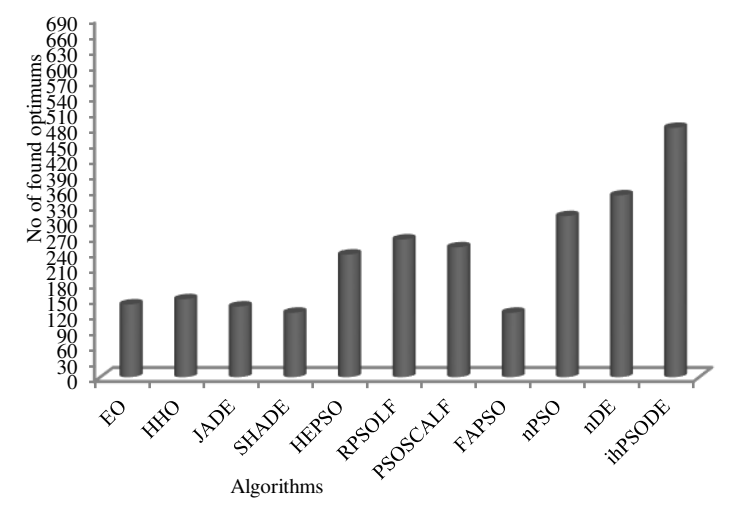

Fig. 5 Global optimal solution out of 690 runs

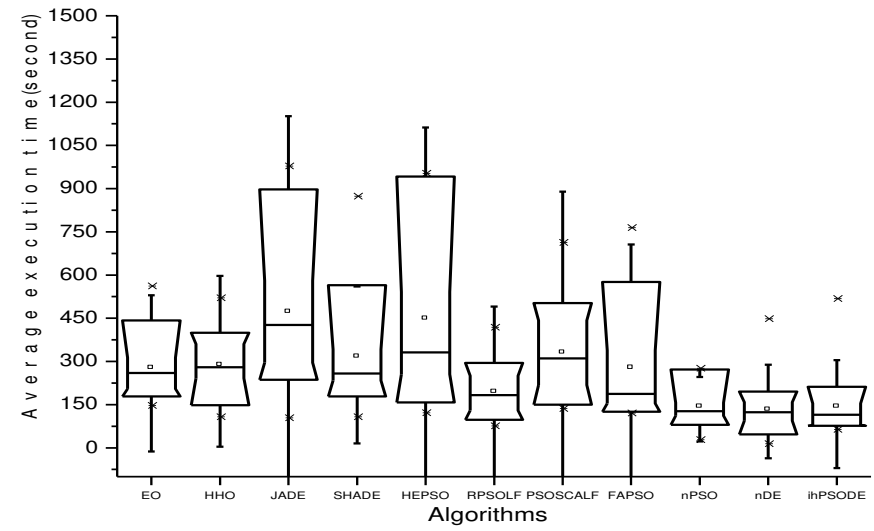

Fig. 6 Processing times of algorithms for $\mathrm{cubf}_{\mathrm{s}}$

In general, it can be decided that the performance of presented algorithms is superior to or at least equal to other intelligent optimization algorithms (classical, PSO, DE and Hybrid variants) on most test functions. In conclusion, presented algorithms (nPSO, nDE, and $\mathrm{i} h \mathrm{PSODE}$ ) can be considered as an effective and efficient method.

\section{Presented algorithms for CEED problem}

To further investigate the feasibility of presented algorithms (nPSO, nDE, and i $h$ PSODE) in real-life problems, two large scale power engineering optimization problem (ELD and CEED) are considered here. These problem include 3 test systems (3, 6 and 40-unit test system) of ELD and 3 test systems (3, 10 and 40-unit test system) of CEED problem. The obtained best solutions are utilized to evaluate the feasibility of different algorithms.

\section{Unit Test Systems}

\begin{tabular}{lll}
\hline Problem & Unit Test Systems $\left(\mathrm{UTS}_{\mathrm{ys}}\right)$ & Description \\
\hline \multirow{3}{*}{ ELD } & $\begin{array}{l}\text { UTS-1 (3-unit test system) } \\
\text { (Hardiansyah et al. 2013) }\end{array}$ & it involves load demand of 300MW. \\
& $\begin{array}{l}\text { UTS-2 (6-unit test system) } \\
\text { (Serapiao 2013) }\end{array}$ & it involves 700MW total load demands. \\
& UTS-3 (40-unit test system) & \\
& (Hardiansyah 2013) & it consist valve loading point effect and \\
& UTS-4 (3-unit test system) & involves load demand of 10500MW. \\
& (Devi and Krishna 2008) & it consider emission impact and involves 400 \\
CEED & UTS-5 (10-unit test system) & MW and 500 MW load demand as well. \\
& (Basu 2011) & it consider valve point effects and involves \\
& UTS-6 (40-unit test system) & 2000MW total load demand. \\
& (Basu 2011) & it consists of non-smooth fuel cost and \\
& & emission functions and involves 10500MW \\
\end{tabular}

\section{Computational Steps of i $h$ PSODE for CEED problem}

The steps of ihPSODE for solving CEED problem are given as below:

1-step Read the $P_{D}$ (Power Demand)

2-step Compute $h$ (price penalty factor)

3-step $\quad t$ (iteration) $=1$

4-step Generate initial population vector of real power generator (based on prohibited zone and ramp limit constraints)

5-step Evaluate the fitness function using equation (5)

6-step Sort the population (as per fitness function value)

7-step Apply nPSO (in best half population) 
8-step Apply nDE (in offspring generated by nPSO)

9-step Merge the population produced by nPSO and $\mathrm{nDE}$

10-step Stop if reaching the termination condition otherwise repeats the steps from 5.

11-step Print the results (generator schedule, minimized operating cost, corresponding fuel cost, and emission output)

\subsection{Results and discussions}

The results of presented hybrid algorithms (nPSO, nDE and i $h$ PSODE) are compared with CDE-SQP (Coelho and Mariani 2006), PPSO (Chen and Yeh 2006), APPSO (Chen and Yeh 2006), SFL (Serapião 2009), BFO (Serapião 2009), CCPSO (Park et al. 2010), SOMA (Coelho and Mariani 2010), CSOMA (Coelho and Mariani 2010), DE/BBO (Bhattacharya and Chattopadhyay 2010), EDA/DE (Wang et al. 2010), ARCGA (Amjady and Rad 2010), TSAGA (Subbaraj et al. 2011), MODE (Basu 2011), NSGAII (Basu 2011), PDE (Basu 2011), SPEA-2 (Basu 2011), GA (Kumar and Alwarsamy 2012), PSO (Kumar and Alwarsamy 2012), GSA (Güvenç et al. 2012), ABC_PSO (Manteaw and Odero 2012), DE (Kumar and Alwarsamy 2012), QP (Hardiansyah et al. 2013), EMOCA (Zhang et al. 2013), SA (Hardiansyah et al. 2013), CS (Serapiao 2013), DHS (Wang and Li 2013), ABC (Serapiao 2013), FA (Serapiao 2013), MPSO (Hardiansyah 2013), MABC/D/Cat (Secui 2015) and MABC/D/Log (Secui 2015), WOA (Mirjalili and Lewis 2016), FPA (Abdelaziz et al. 2016), GA-WOA (Edwin Selva Rex et al. 2019), $\beta$ GWO (Betar et al. 2020), on ELD and CEED test systems.

For clearness, stopping criteria (500 iterations) population size (30) and independent run (25) of presented algorithms is taken same as relative algorithms. Rest parameter of proposed methods are same as above. The simulations result of presented algorithms with other comparative algorithms are listed in Table 5 (for UTS-1), Table 6 (for UTS-2), Table 7 (for UTS-3), Table 8 (for UTS-4), Table 9 (for UTS5) and Table 10 (for UTS-6).

Table 5 Simulation results for UTS-1

\begin{tabular}{llllllllll}
\hline Outputs (MW) & QP & SA & GA & PSO & DE & FPA & nPSO & nDE & i $h$ PSODE \\
\hline $\mathrm{P}^{1}$ & 207.6799 & 207.6336 & 208.99 & 209.001 & 207.637 & 207.6316 & 204.3651 & 205.1546 & 205.3596 \\
$\mathrm{P}^{2}$ & 87.4010 & 87.2867 & 86.0041 & 85.92 & 87.2833 & 87.2886 & 81.3969 & 82.2746 & 82.0584 \\
$\mathrm{P}^{3}$ & 15.0000 & 15.0000 & 15.4163 & 15.0000 & 15.0000 & 15.0000 & 14.238 & 12.5708 & 14.582 \\
$\mathrm{P}_{\mathrm{L}}$ & 10.0808 & 9.9203 & 10.4099 & 9.9833 & 9.9203 & 9.9202 & 8.6788 & 8.7645 & 7.7655 \\
$\sum_{\mathrm{Cost}} P_{i}$ & 310.0808 & 309.9203 & 310.4099 & 309.9211 & 309.9203 & 309.9202 & 308.6788 & 308.7645 & 309.7655 \\
CPU(s) & 3621.50 & 3619.76 & 3624.28 & 3621.75 & 3619.8 & 3619.75 & 3619.88 & 3619.55 & 3619.45 \\
\hline
\end{tabular}

Table 6 Simulation results for UTS-2

\begin{tabular}{lllllllllll}
\hline Outputs (MW) & CS & ABC & FA & PSO & SFL & BFO & FPA & nPSO & nDE & i $h$ PSODE \\
\hline $\mathrm{P}^{1}$ & 324.113 & 323.043 & 293.312 & 288.653 & 287.392 & 222.260 & 323.995 & 321.2581 & 321.001 & 321.995 \\
$\mathrm{P}^{2}$ & 76.859 & 54.965 & 79.546 & 82.753 & 67.637 & 58.777 & 76.846 & 73.4416 & 74.846 & 74.322 \\
$\mathrm{P}^{3}$ & 158.094 & 147.354 & 123.334 & 132.988 & 140.933 & 150.395 & 158.20 & 154.3169 & 155.4747 & 153.1017 \\
$\mathrm{P}^{4}$ & 50 & 50 & 69.7 & 50 & 98.357 & 106.963 & 50 & 50.0000 & 50.0000 & 50.0000 \\
$\mathrm{P}^{5}$ & 51.963 & 85.815 & 79.546 & 99.565 & 64.052 & 101.601 & 51.983 & 50.9834 & 50.1583 & 50.5813 \\
$\mathrm{P}^{6}$ & 50 & 50.233 & 63.778 & 57.768 & 53.15 & 72.559 & 50 & 50.0000 & 48.5200 & 50.0000 \\
$\mathrm{P}_{\mathrm{L}}$ & 11.03 & 11.4 & 11.44 & 11.73 & 11.59 & 11.73 & 11.024 & 11.0122 & 11.0124 & 11.0245 \\
$\sum_{\mathrm{Cost}} P_{i}$ & 711.03 & 711.4 & 711.44 & 711.73 & 711.59 & 711.73 & 711.024 & 711.0122 & 711.0124 & 711.0245 \\
CPU(s) & 8356.06 & 8372.27 & 8388.45 & 8401.45 & 8419.78 & 8428.69 & 8356.05 & 8368.8401 & 8359.2141 & 8356.1545 \\
\hline
\end{tabular}

Table 7 Simulation results for UTS-3

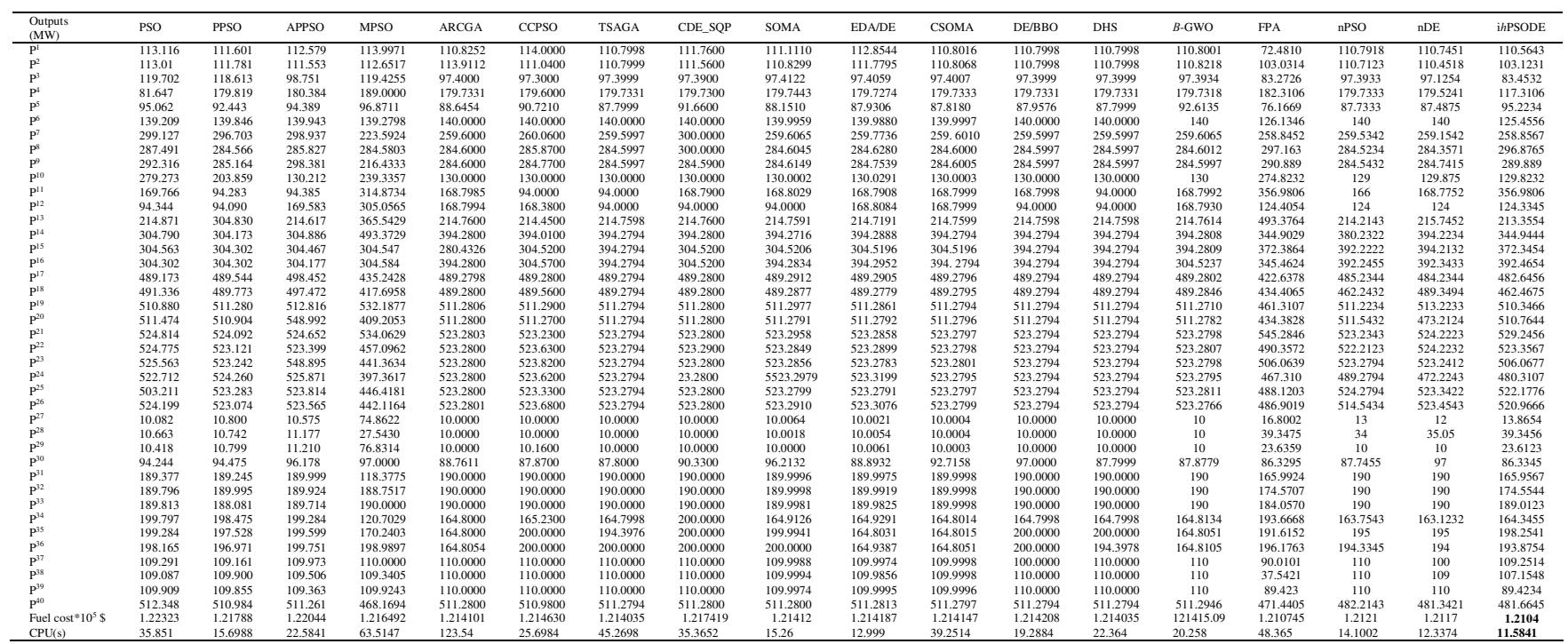


Table 8 Simulation results for UTS-4

\begin{tabular}{|c|c|c|c|c|c|c|c|c|c|c|}
\hline$P_{D}$ & $h$ & Power outputs (MW) & GA & PSO & FPA & WOA & GA-WOA & nPSO & $\mathrm{nDE}$ & i $h$ PSODE \\
\hline \multirow{8}{*}{400 (MW) } & \multirow{8}{*}{43.55981} & $\mathrm{P}^{\mathrm{I}}$ & 102.617 & 102.612 & 102.4468 & 102.4887 & 102.5355 & 101.6157 & 101.0747 & 101.6155 \\
\hline & & $\mathrm{P}^{2}$ & 153.825 & 153.809 & 153.8341 & 153.8043 & 153.7200 & 151.2972 & 151.8354 & 151.2563 \\
\hline & & $\mathrm{P}^{3}$ & 151.011 & 150.991 & 151.1321 & 151.1278 & 151.1046 & 147.0871 & 147.0899 & 147.1282 \\
\hline & & $P_{L}$ & 7.41324 & 7.41173 & 7.4126 & 7.4208 & 7.4182 & 7.4184 & 7.4122 & 7.4102 \\
\hline & & Fuel cost (\$) & 20840.1 & 20838.3 & 20838.1 & 20838 & 20836 & 20839.4512 & 20838.1544 & 20837.2154 \\
\hline & & Emission (kg) & 200.256 & 200.221 & 200.2238 & 200.2316 & 200.1748 & 200.2210 & 200.2204 & 200.2193 \\
\hline & & Total cost $(\$)$ & 29563.2 & 29559.9 & 29559.81 & 29560 & 29556 & 29559.6854 & 29558.6500 & 29557.3589 \\
\hline & & $\mathrm{CPU}(\mathrm{s})$ & 0.282 & 0.235 & 0.175 & 0.297 & 0.783327 & 0.212 & 0.189 & 0.115 \\
\hline \multirow{8}{*}{$500(\mathrm{MW})$} & \multirow{8}{*}{44.07915} & P1 & 128.997 & 128.984 & 128.8074 & 128.5434 & 128.5344 & 125.8785 & 125.2001 & 125.997 \\
\hline & & P2 & 192.683 & 192.645 & 192.5906 & 192.6543 & 192.7451 & 187.0074 & 186.6855 & 185.5519 \\
\hline & & P3 & 190.11 & 190.063 & 190.2958 & 190.2876 & 190.2784 & 187.1141 & 188.1144 & 188.4511 \\
\hline & & $\mathrm{P}_{\mathrm{L}}$ & 11.6964 & 11.6919 & 11.6938 & 11.6954 & 11.6811 & 11.6964 & 11.7854 & 11.6964 \\
\hline & & Fuel cost (\$) & 25499.4 & 25495 & 25494.7 & 25495.744 & 25494.568 & 25493.4145 & 25495.1522 & 25499.4 \\
\hline & & Emission $(\mathrm{kg})$ & 311.273 & 311.15 & 311.155 & 311.165 & 311.1485 & 311.1152 & 311.1093 & 311.1021 \\
\hline & & Total cost(\$) & 39220.1 & 39210.2 & 39210.15 & 39219.210 & 39218.541 & 39209.125 & 39210.168 & 39208.181 \\
\hline & & CPU(s) & 0.172 & 0.156 & 0.126 & 0.183 & 0.177 & 0.162 & 0.154 & 0.122 \\
\hline
\end{tabular}

Table 9 Simulation results for UTS-5

\begin{tabular}{|c|c|c|c|c|c|c|c|c|c|c|c|}
\hline Outputs(MW) & MODE & NSGAII & PDE & SPEA-2 & GSA & ABC_PSO & EMOCA & FPA & nPSO & $\mathrm{nDE}$ & ihPSODE \\
\hline $\mathrm{P}^{1}$ & 54.9487 & 51.9515 & 54.9853 & 52.9761 & 54.9992 & 55 & 55 & 53.188 & 52.9215 & 52.1522 & 52.9755 \\
\hline $\mathrm{P}^{2}$ & 74.5821 & 67.2584 & 79.3803 & 72.813 & 79.9586 & 80 & 80 & 79.975 & 72.8131 & 72.8131 & 72.8541 \\
\hline $\mathrm{P}^{3}$ & 79.4294 & 73.6879 & 83.9842 & 78.1128 & 79.4341 & 81.14 & 83.5594 & 78.105 & 78.1122 & 78.2511 & 78.1185 \\
\hline $\mathrm{P}^{4}$ & 80.6875 & 91.3554 & 86.5942 & 83.6088 & 85.0000 & 84.216 & 84.6031 & 97.119 & 83.6785 & 83.5412 & 83.6085 \\
\hline $\mathrm{P}^{5}$ & 136.8551 & 134.0522 & 144.4386 & 137.2432 & 142.1063 & 138.3377 & 146.5632 & 152.74 & 137.2455 & 137.1542 & 137.1522 \\
\hline $\mathrm{P}^{6}$ & 172.6393 & 174.9504 & 165.7756 & 172.9188 & 166.5670 & 167.5086 & 169.2481 & 163.08 & 172.9145 & 172.8214 & 172.9128 \\
\hline $\mathrm{P}^{7}$ & 283.8233 & 289.4350 & 283.2122 & 287.2023 & 292.8749 & 296.8338 & 300 & 258.61 & 287.2154 & 287.2514 & 287.2154 \\
\hline $\mathrm{P}^{8}$ & 316.3407 & 314.0556 & 312.7709 & 326.4023 & 313.2387 & 311.5824 & 317.3496 & 302.22 & 326.4153 & 324.4985 & 326.4469 \\
\hline $\mathrm{P}^{9}$ & 448.5923 & 455.6978 & 440.1135 & 448.8814 & 441.1775 & 420.3363 & 412.9183 & 433.21 & 448.8824 & 449.8785 & 448.8258 \\
\hline $\mathrm{P}^{10}$ & 436.4287 & 431.8054 & 432.6783 & 423.9025 & 428.6306 & 449.1598 & 434.3133 & 466.07 & 423.9045 & 425.9125 & 423.9025 \\
\hline Fuel cost*10 $10^{5}(\$)$ & 1.13484 & 1.13539 & 1.1351 & 1.1352 & 1.1349 & 1.1342 & 1.13445 & 1.1337 & 1.1351 & 1.1349 & 1.1335 \\
\hline Emission (lb) & 4124.9 & 4130.2 & 4111.4 & 4109.1 & 4111.4 & 4120.1 & 4113.98 & 3997.7 & 3998.7452 & 3996.0222 & 3994.2514 \\
\hline Loses (MW) & 84.33 & 84.25 & 83.9 & 84.1 & 83.9869 & 84.1736 & 83.56 & 84.3 & 84.103 & 84.274 & 84.012 \\
\hline$h$ & 8.2877 & 8.2398 & 8.9955 & 8.23567 & 8.54322 & 8.96788 & 8.99078 & 8.99967 & 8. 22284 & 8.21887 & 8. 21255 \\
\hline $\mathrm{CPU}(\mathrm{s})$ & 3.82 & 6.02 & 4.23 & 7.53 & 3.68 & 3.65 & 2.90 & 2.23 & 3.56 & 3.25 & 2.22 \\
\hline
\end{tabular}

Table 10 Simulation results for UTS-6

\begin{tabular}{|c|c|c|c|c|c|c|c|c|c|c|c|c|c|}
\hline Outputs (MW) & MODE & PDE & NSGA-II & SPEA-2 & GSA & MABC/D/Cat & WOA & MABC/D/Log & FPA & GA-WOA & nPSO & $\mathrm{nDE}$ & i $h$ PSODE \\
\hline $\mathrm{P}^{1}$ & 113.5295 & 112.1549 & 113.8685 & 113.9694 & 113.9989 & 110.7998 & 103.5252 & 110.7998 & 43.405 & 103.42601 & 113.1254 & 113.2581 & 113.5214 \\
\hline $\mathrm{P}^{2}$ & 114 & 113.9431 & 113.6381 & 114 & 113.9896 & 110.7998 & 102.8142 & 110.7998 & 113.95 & 102.9235 & 113.4851 & 113.7854 & 113.9158 \\
\hline $\mathrm{P}^{3}$ & 120 & 120 & 120 & 119.8719 & 119.9995 & 97.3999 & 93.399 & 97.3999 & 105.86 & 92.01605 & 119.9999 & 119.1524 & 119.5562 \\
\hline $\mathrm{P}^{4}$ & 179.8015 & 180.2647 & 180.7887 & 179.9284 & 179.7857 & 174.5504 & 181.2391 & 174.5486 & 169.65 & 183.828 & 179.8547 & 179.3584 & 179.7211 \\
\hline $\mathrm{P}^{5}$ & 96.7716 & 97 & 97 & 97 & 97 & 87.7999 & 88.6654 & 97 & 96.659 & 84.01458 & 97.0000 & 97.2514 & 97.0000 \\
\hline $\mathrm{P}^{6}$ & 139.2760 & 140 & 140 & 139.2721 & 139.0128 & 105.3999 & 123.93 & 105.3999 & 139.02 & 123.9824 & 139.1145 & 139.1542 & 139.1452 \\
\hline $\mathrm{P}^{7}$ & 300 & 299.8829 & 300 & 300 & 299.9885 & 259.5996 & 258.589 & 259.5996 & 273.28 & 259.9376 & 299.7896 & 299.7854 & 299.9885 \\
\hline $\mathrm{P}^{8}$ & 298.9193 & 300 & 299.0084 & 298.2706 & 300 & 284.5996 & 282.982 & 284.5996 & 285.17 & 294.4696 & 300.0000 & 300.0000 & 300.0000 \\
\hline $\mathrm{P}^{9}$ & 290.7737 & 289.8915 & 288.8890 & 290.5228 & 296.2025 & 284.5996 & 288.395 & 284.5996 & 241.96 & 289.805 & 296.2845 & 296.7541 & 296.2514 \\
\hline $\mathrm{P}^{10}$ & 130.9025 & 130.5725 & 131.6132 & 131.4832 & 130.3850 & 130 & 221.951 & 130 & 131.26 & 248.272 & 130.358 & 130.3581 & 130.3854 \\
\hline $\mathrm{P}^{11}$ & 244.7349 & 244.1003 & 246.5128 & 244.6704 & 245.4775 & 318.1921 & 188.5985 & 318.2129 & 312.13 & 198.1655 & 245.4859 & 245.7854 & 245.1526 \\
\hline $\mathrm{P}^{12}$ & 317.8218 & 318.2840 & 318.8748 & 317.2003 & 318.2101 & 243.5996 & 128.7994 & 243.5996 & 362.58 & 123.955 & 318.2052 & 318.7845 & 318.2055 \\
\hline $\mathrm{P}^{13}$ & 395.3846 & 394.7833 & 395.7224 & 394.7357 & 394.6257 & 394.2793 & 451.763 & 394.2793 & 346.24 & 441.354 & 394.6025 & 394.6985 & 394.6569 \\
\hline $\mathrm{P}^{14}$ & 394.4692 & 394.2187 & 394.1369 & 394.6223 & 395.2016 & 394.2793 & 394.28 & 394.2793 & 306.06 & 398.9032 & 395.2154 & 395.1458 & 395.2115 \\
\hline $\mathrm{P}^{15}$ & 305.8104 & 305.9616 & 305.5781 & 304.7271 & 306.0014 & 394.2793 & 354.081 & 394.2793 & 358.78 & 382.0418 & 306.0015 & 306.0008 & 306.0078 \\
\hline $\mathrm{P}^{16}$ & 394.8229 & 394.1321 & 394.6968 & 394.7289 & 395.1005 & 394.2793 & 334.28 & 394.2793 & 260.68 & 342.2716 & 395.1007 & 395.1485 & 395.1005 \\
\hline $\mathrm{P}^{17}$ & 487.9872 & 489.3040 & 489.4234 & 487.9857 & 489.2569 & 399.5195 & 429.2798 & 399.5195 & 415.19 & 469.7822 & 489.2567 & 489.2569 & 489.3588 \\
\hline $\mathrm{P}^{18}$ & 489.1751 & 489.6419 & 488.2701 & 488.5321 & 488.7598 & 399.5195 & 489.128 & 399.5195 & 423.94 & 445.1378 & 489.1752 & 489.1751 & 489.1158 \\
\hline $\mathrm{P}^{19}$ & 500.5265 & 499.9835 & 500.8 & 501.1683 & 499.2320 & 506.1985 & 451.2306 & 506.1716 & 549.12 & 462.328 & 500.5268 & 500.7854 & 500.5562 \\
\hline$P^{20}$ & 457.0072 & 455.4160 & 455.2006 & 456.4324 & 455.2821 & 506.1985 & 461.88 & 506.2206 & 496.7 & 482.812 & 457.0021 & 457.3598 & 457.0152 \\
\hline $\mathrm{P}^{21}$ & 434.6068 & 435.2845 & 434.6639 & 434.7887 & 433.4520 & 514.1472 & 523.2803 & 514.1105 & 539.17 & 513.198 & 434.6845 & 434.2541 & 434.6085 \\
\hline $\mathrm{P}^{22}$ & 434.5310 & 433.7311 & 434.15 & 434.3937 & 433.8125 & 514.1455 & 485.28 & 514.1472 & 546.46 & 499.9768 & 434.5584 & 434.2541 & 434.5521 \\
\hline $\mathrm{P}^{23}$ & 444.6732 & 446.2496 & 445.8385 & 445.0772 & 445.5136 & 514.5237 & 521.458 & 514.5664 & 540.06 & 528.032 & 444.6485 & 444.6732 & 444.6895 \\
\hline $\mathrm{P}^{24}$ & 452.0332 & 451.8828 & 450.7509 & 451.8970 & 452.0547 & 514.5386 & 493.311 & 514.4868 & 514.5 & 469.3765 & 452.4851 & 445.0732 & 452.4851 \\
\hline $\mathrm{P}^{25}$ & 492.7831 & 493.2259 & 491.2745 & 492.3946 & 492.8864 & 433.5196 & 523.28 & 433.5195 & 453.46 & 499.1242 & 492.7877 & 492.7131 & 492.2514 \\
\hline $\mathrm{P}^{26}$ & 436.3347 & 434.7492 & 436.3418 & 436.9926 & 433.3695 & 433.5195 & 481.2801 & 433.5196 & 517.31 & 499.8282 & 436.3377 & 438.3847 & 436.3589 \\
\hline $\mathrm{P}^{27}$ & 10 & 11.8064 & 11.2457 & 10.7784 & 10.0026 & 10 & 12.33 & 10 & 14.881 & 15.7668 & 10.0000 & 10.0000 & 10.0000 \\
\hline $\mathrm{P}^{28}$ & 10.3901 & 10.7536 & 10 & 10.2955 & 10.0246 & 10 & 21 & 10 & 18.79 & 18.06882 & 10.3901 & 10.3971 & 10.3988 \\
\hline $\mathrm{P}^{29}$ & 12.3149 & 10.3053 & 12.0714 & 13.7018 & 10.0125 & 10 & 14.17 & 10 & 26.611 & 12.98864 & 12.3158 & 12.1349 & 12.3188 \\
\hline $\mathrm{P}^{30}$ & 96.9050 & 97 & 97 & 96.2431 & 96.9125 & 97 & 88.7611 & 87.8042 & 59.58 & 88.05685 & 96.1548 & 96.9108 & 96.9888 \\
\hline $\mathrm{P}^{31}$ & 189.7727 & 189.4826 & 190.0000 & 189.4826 & 190.0000 & 189.9689 & 190 & 159.733 & 183.48 & 162.1605 & 189.7154 & 189.7727 & 184.7757 \\
\hline $\mathrm{P}^{32}$ & 174.2324 & 175.3065 & 174.7971 & 174.2163 & 175 & 159.733 & 190 & 159.7331 & 183.39 & 188.2118 & 173.7524 & 174.2898 & 174.2154 \\
\hline $\mathrm{P}^{33}$ & 190 & 190 & 189.2845 & 190 & 189.0181 & 159.733 & 190 & 159.733 & 189.02 & 181.089 & 186.4856 & 189.4589 & 189.1544 \\
\hline $\mathrm{P}^{34}$ & 199.6506 & 200 & 200 & 200 & 200 & 200 & 161.834 & 200 & 198.73 & 174.1796 & 199.3355 & 199.7895 & 199.4851 \\
\hline $\mathrm{P}^{35}$ & 199.8662 & 200 & 199.9138 & 200 & 200 & 200 & 163.891 & 200 & 198.77 & 195.0134 & 199.1548 & 199.1485 & 199.1593 \\
\hline $\mathrm{P}^{36}$ & 200 & 200 & 199.5066 & 200 & 199.9978 & 200 & 169.8054 & 200 & 182.23 & 194.884 & 198.6348 & 197.8952 & 199.0541 \\
\hline $\mathrm{P}^{37}$ & 110 & 109.9412 & 108.3061 & 110 & 109.9969 & 89.1141 & 109.389 & 89.1141 & 39.673 & 98.4301 & 110.0000 & 110.0000 & 110.0000 \\
\hline $\mathrm{P}^{38}$ & 109.9454 & 109.8823 & 110 & 109.6912 & 109.0126 & 89.1141 & 110.12 & 89.1141 & 81.596 & 109.5671 & 109.1648 & 109.9458 & 109.5841 \\
\hline $\mathrm{P}^{39}$ & 108.1786 & 108.9686 & 109.7899 & 108.5560 & 109.4560 & 89.1141 & 110.91 & 89.1141 & 42.96 & 108.8450 & 108.6586 & 108.1214 & 108.1658 \\
\hline $\mathrm{P}^{40}$ & 422.0628 & 421.3778 & 421.3778 & 421.5609 & 421.8521 & 421.9987 & 511.28 & 506.1879 & 537.17 & 505.7928 & 421.1528 & 421.7845 & 421.8855 \\
\hline ost*10 $10^{5}(\$)$ & 1.2579 & 1.2573 & 1.2583 & 1.2581 & 1.2578 & 1.2449090 & 1.23644 & 1.24491161 & 1.23170 & 1.22862 & 1.2365 & 1.2236 & 1.2225 \\
\hline Emission* $10^{5}$ (ton) & 2.1119 & 2.1177 & 2.1095 & 2.1110 & 2.1093 & 2.5656026 & 2.1324 & 2.56560267 & 2.0846 & 2.06850 & 2.1094 & 2.1025 & 2.0985 \\
\hline CPU(s) & 5.39 & 6.15 & 7.32 & 8.57 & 5.69 & 6.25 & 5.02 & 5.65 & 4.92 & 5.51 & 4.55 & 4.25 & 3.25 \\
\hline
\end{tabular}

As specified in these tables the best cost created by- (i) presented nPSO for UTS-1, UTS-2 and UTS-3 of ELD problems are $3619.88(\$ / \mathrm{hr}), 8368.8401(\$ / \mathrm{hr})$ and $1.2121 \times 10^{5}(\$ / \mathrm{hr})$ respectively and for UTS-4 (400 and 500 MW load demand), UTS-5 and UTS-6 of CEED problems are $29559.6854(\$ / \mathrm{hr})$, $39209.125(\$ / \mathrm{hr}), 1.1351 \times 10^{5}(\$ / \mathrm{hr})$ and $1.2365 \times 10^{5}(\$ / \mathrm{hr})$ separately. (ii) proposed nDE for UTS-1, UTS2 and UTS-3 of ELD problems are 3619.55(\$/hr), 8359.2141(\$/hr), and $1.2117 \times 10^{5}(\$ / \mathrm{hr})$ respectively and for UTS-4 (400 and 500 MW load demand), UTS-5 and UTS-6 of CEED problems are 29558.6500 $(\$ / \mathrm{hr}), 39210.168(\$ / \mathrm{hr}), 1.1349 \times 10^{5}(\$ / \mathrm{hr})$ and $1.2236 \times 10^{5}(\$ / \mathrm{hr})$ correspondingly. (iii) proposed ihPSODE for UTS-1, UTS-2, and UTS-3 of ELD problems are 3619.45(\$/hr), 8356.1545(\$/hr) and 
$1.2104 \times 10^{5}(\$ / \mathrm{hr})$ respectively and for UTS-4 (400 and $500 \mathrm{MW}$ load demand), UTS-5 and UTS-6 of CEED problems are $29557.3589(\$ / \mathrm{hr}), 39208.181(\$ / \mathrm{hr}), 1.1335 \times 10^{5}(\$ / \mathrm{hr})$ and $1.2225 \times 10^{5}(\$ / \mathrm{hr})$ individually.

According to the reported cost results, the proposed nPSO, nDE, and i $h$ PSODE algorithms have the lowest fuel cost and emission when compared to other compared algorithms for all unit test systems. Furthermore, CPU average times for each unit test system are noted in the associated tables, demonstrating that the proposed algorithms produce better solutions in less time than others. As a result, the proposed algorithms outperform and outlast other compared algorithms in terms of reducing total cost in the shortest amount of time. This indicates that the presented algorithm has higher reliability/robustness, stability and convergence when compared to other algorithms.

The convergence curves of presented and other algorithms are plotted in Fig. 7(a-g) for UTS-1, UTS-2, UTS-3, UTS-4, UTS-5 and UTS-6 in terms of total cost versus iterations. These figures show that presented algorithms (nPSO, nDE, and ihPSODE) has better convergence performance.

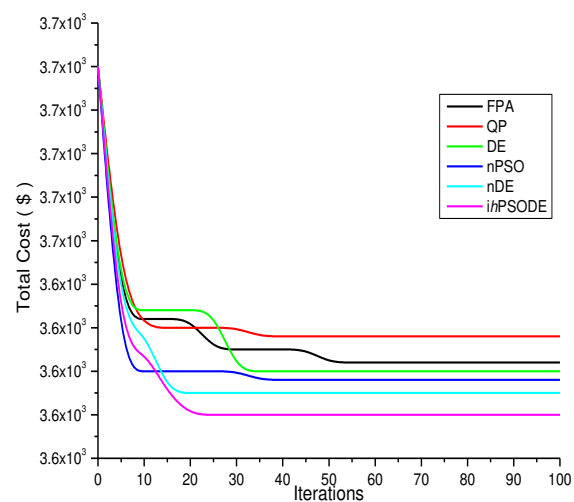

(a) UTS-1

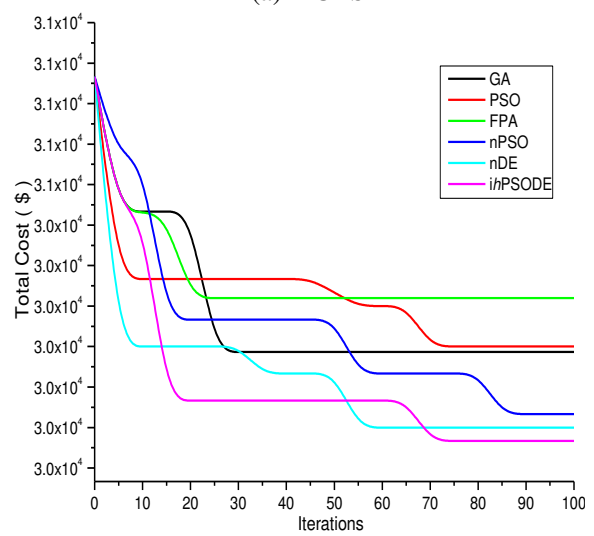

(d) UTS-4(400 MW)

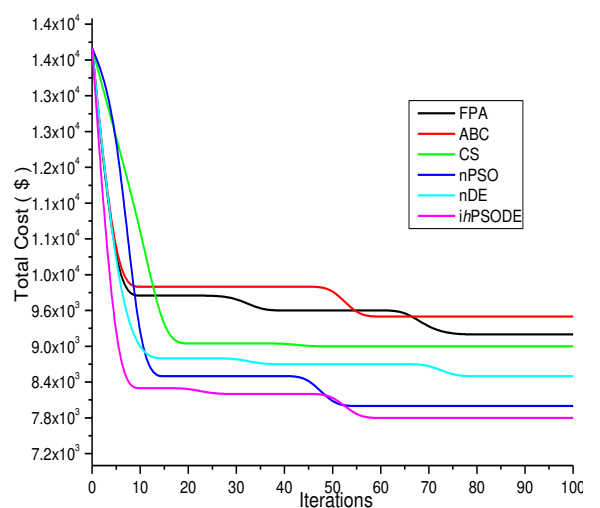

(b) UTS-2

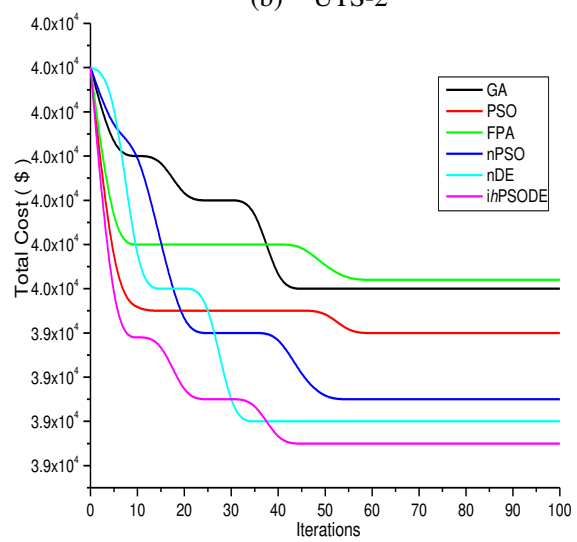

(e) UTS-4 (500MW)

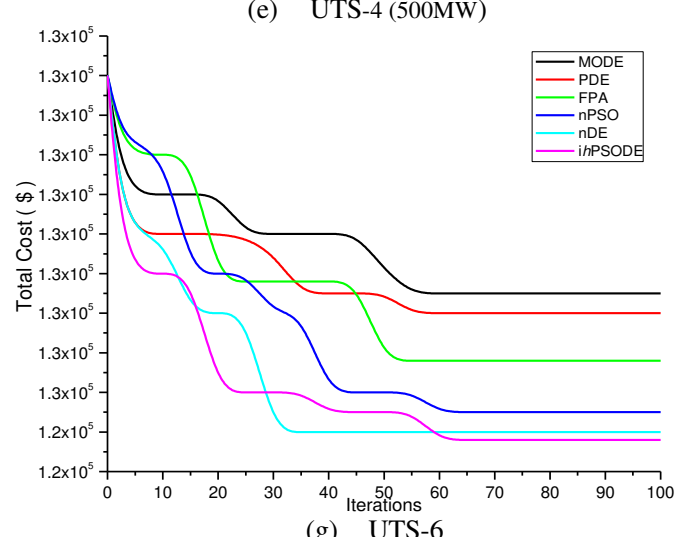

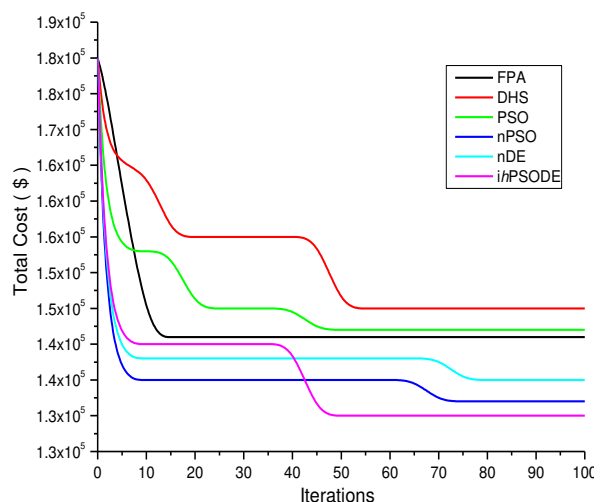

(c) UTS-3

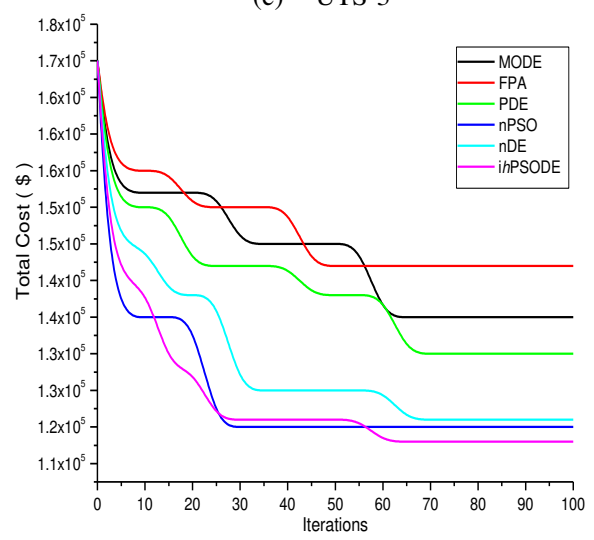

(f) UTS-5

Fig. 7(a-g) Cost convergence characteristic for different test systems

Moreover, fuel cost variations for all test systems presented in Fig. 8 (a-g). It shows and confirmed effectiveness of the proposed $\mathrm{nPSO}, \mathrm{nDE}$ and $\mathrm{i} h \mathrm{PSODE}$ for decreasing the fuel cost. Also, these figures demonstrate that the supremacy of the presented algorithm in attaining minimum fuel cost compared to others with different demands. Therefore, presented algorithms are economically competent. At large, it can be stating that (from the all above result investigation) presented algorithms (nPSO, $\mathrm{nDE}$ and 
ihPSODE) are performing better and/or similar with others. Still, between three presented algorithms hybrid algorithm i.e. ihPSODE have greater capability.

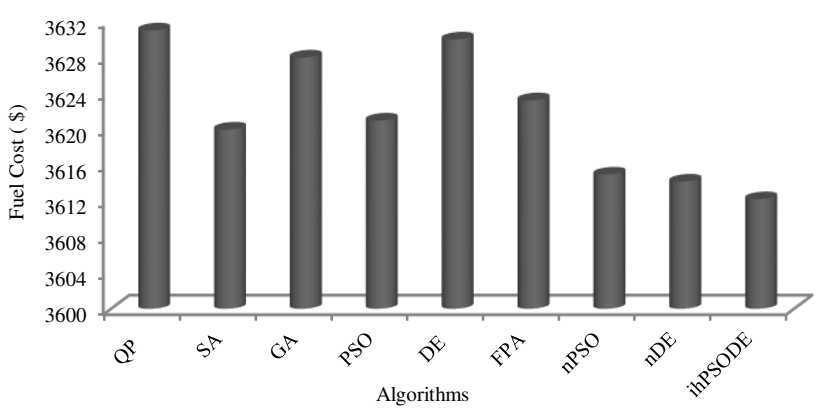

(a). UTS-1

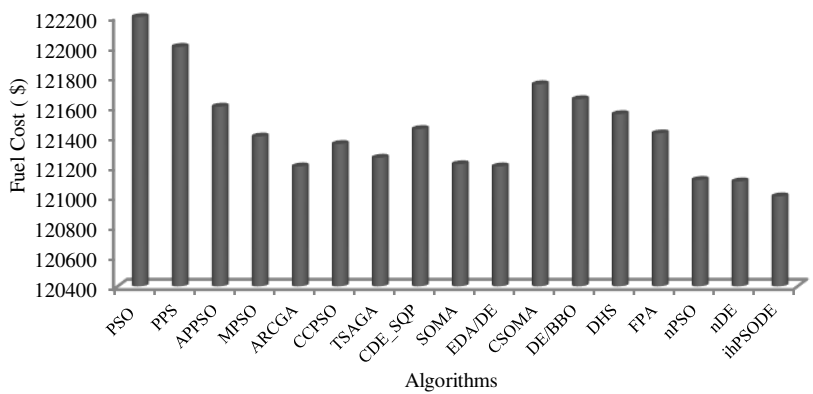

(c). UTS-3

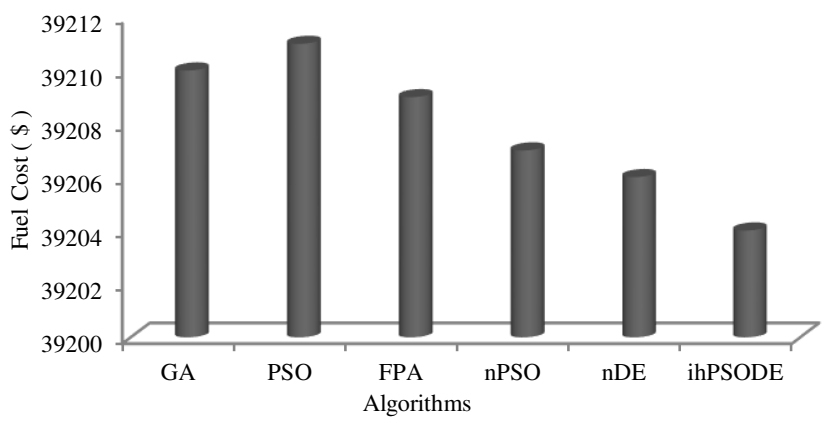

(e). UTS-4 (500 MW)

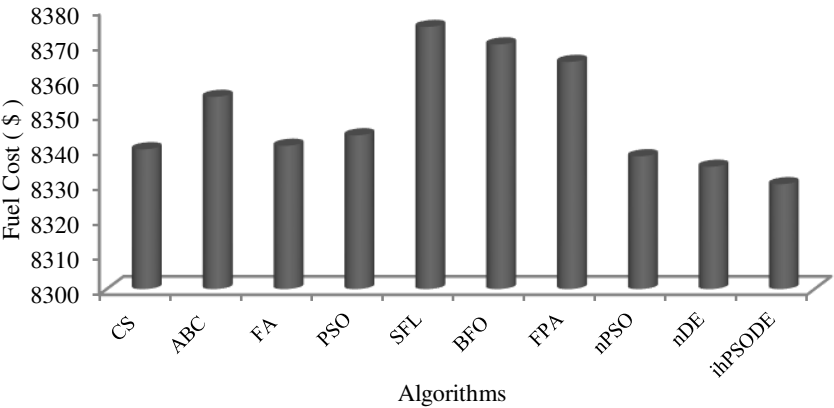

(b). UTS-2

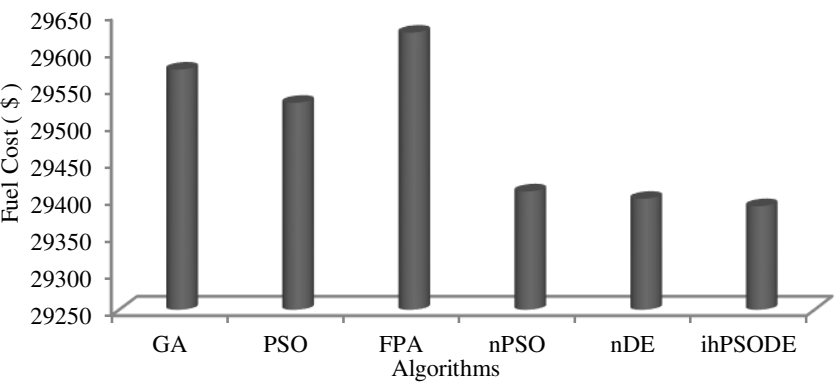

(d). UTS-4 (400 MW)

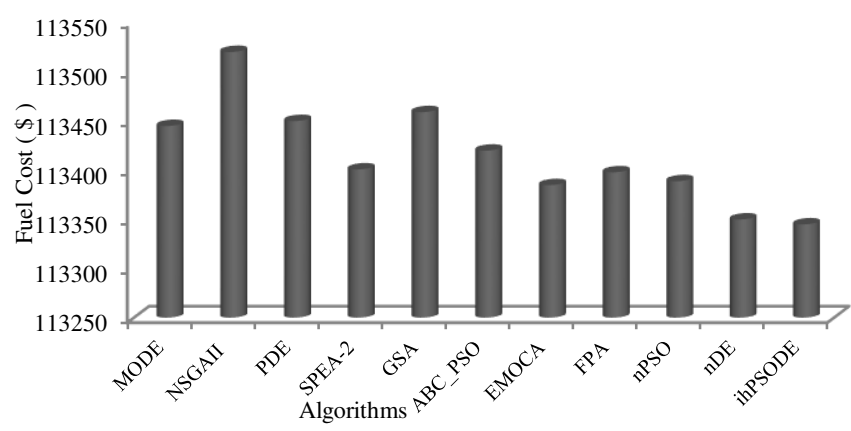

(f). UTS-5

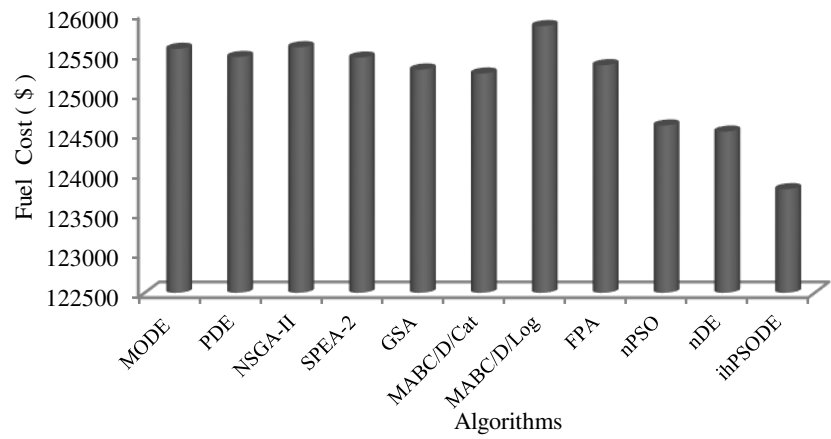

(g). UTS-6

Fig. 8(a-g) Fuel cost variations for different unit test systems

\subsection{Complexity analysis}

In this section some complexity examination of the presented algorithms is specified as below.

\section{i). time complexity}

Presented i $h$ PSODE has the following time complexity (according to the steps).

a). $n p$-population initialization needs $O(n p . D)$ time.

b). evaluation and sorting (as per fitness function values) population wants $O\left(t_{\max } \times n p\right)$ time.

c). partition of population $\left(\mathrm{pop}_{1}\right.$ and $\left.\mathrm{pop}_{2}\right)$ requires $O\left(t_{\max } \times n p\right)$ time.

d). calculation of $p o p_{1}\left(\right.$ by nDE) and $p o p_{2}$ (by nPSO) takes $O\left(t_{\max } \times \frac{n p}{2} \times \frac{n p}{2}\right)=O\left(t_{\max } \times \frac{n p^{2}}{4}\right)$ time. 
e). population integration and execution of algorithm involves $O\left(t_{\max } \times n p \times n p\right)=O\left(t_{\max } \times n p^{2}\right)$ time.

So, for maximum number of iterations the total time complexity of i $h$ PSODE is-

$$
O(n p . D)+O\left(t_{\max } \times n p\right)+O\left(t_{\max } \times n p\right)+O\left(t_{\max } \times \frac{n p^{2}}{4}\right)+O\left(t_{\max } \times n p^{2}\right)=O\left(t_{\max } \times n p^{2} \times D\right)
$$

\section{ii). space complexity}

The space complexity is the maximum volume of space which is used by presented $\mathrm{i} h \mathrm{PSODE}$ algorithm. Thus, the total space complexity of proposed $\mathrm{i} h \mathrm{PSODE}$ algorithm is $O(\max (n p$, $\left.\left.n p, n p, \frac{n p^{2}}{4}, n p^{2}\right) \times D\right)=O\left(n p^{2} \times D\right)$.

\section{Conclusion with future perspectives}

In this paper, to promote the performance of PSO and DE algorithm, a novel PSO (namely nPSO), novel DE (called nDE) and their innovative hybrid (titled ihPSODE) is presented for solving combined economic and emission dispatch (CEED) problems. Presented nPSO has a new acceleration coefficient, inertia weight and position improve equation (to alleviate the stagnation) as well as $\mathrm{nDE}$ has a new mutation approach and crossover rate (to prevent premature convergence). After population evaluation (in i $h$ PSODE) best half member has been recognized and nPSO employed (which enhanced local and global search capacity) then $\mathrm{nDE}$ (which ensures bring solutions with higher quality), in each iteration process. In addition, because of suitable implementation of nPSO and $\mathrm{nDE}$, particle can learn not only from the globally based individuals, but also from the best individuals of each problem in $i h$ PSODE. Altogether, quality of 'memorizing (by nPSO)' and 'diversity maintaining (by nDE)' brands ihPSODE more robust. Likewise, related novel presented control parameters of nPSO and nDE makes extra support for the success of i $h$ PSODE.

The presented algorithms (nPSO, nDE and i $h \mathrm{PSODE}$ ) have been tested over 23 unconstrained benchmark functions then applied to solve two large scale power engineering optimization problem namely economic load dispatch (ELD) and combined economic emission dispatch (CEED) problem. These problem include 3 test systems (3, 6 and 40-unit test system) of ELD and 3 test systems (3, 10 and 40-unit test system) of CEED problem. The performance of presented methods compared with the classical DE and PSO with their existed variants and hybrids plus other state-of-the-art methods.

The simulation results prove that presented algorithms are more effective than or at least competitive to the compared algorithms in case of unconstrained benchmark functions. Moreover, presented algorithms are successfully used to solve ELD and CEED power system engineering optimization problems. The optimization results confirm that presented algorithms can achieve better solutions than other compared methods in case of power system engineering optimization problems. Therefore, presented algorithms are economically competent. All in all, it can be summarized that the proposed algorithms (nPSO, nDE and $\mathrm{i} h \mathrm{PSODE}$ ) can be seen as an effective algorithm to solve power system engineering optimization problems. Lastly, between three presented algorithms hybrid algorithm i.e. ihPSODE have greater capability.

Furthermore, the presented algorithms do have higher time complexity than some PSO, DE and hybrid variants. The matrix operation evaluation is the primary cause of the presented algorithms' timeconsuming nature. This operation is repeated for each individual on each iteration, which increases the algorithm's running time in some extent. Besides, the presented algorithms may not be appropriate for all complex optimization problems.

Some novel parameters will be designed for the presented nDE, nPSO, and $\mathrm{i} h \mathrm{PSODE}$ as part of our future work for finding more precise solutions and falling time complexity. Finally, this paper is expected to devote in a fruitful analysis i.e. complete mathematical convergence analysis of the presented algorithm which may done in the coming paper with inspecting how to advance the strength for multifaceted optimization problems. 


\section{Acknowledgments}

Authors, heartfelt thanks to the Editor and the Reviewers for their constructive suggestions.

\section{Declaration of competing interest}

There are no conflicts of interest.

\section{Data availability statement}

Authors declare that all data analyzed or generated throughout this study are involved in the submitted paper. Similarly, availability of data and materials are cited in references of the paper. Additionally, the data of this study are available from the corresponding author upon reasonable request wich support the findings. No additional data archiving is required.

\section{References}

Abdelaziz AY, Ali ES, Abd Elazim SM (2016) Combined economic and emission dispatch solution using Flower Pollination Algorithm. Electrical Power and Energy Systems 80:264-274

Ajayi O, Heymann R (2021) Day-Ahead Combined Economic and Emission Dispatch with Spinning Reserve Consideration using Moth Swarm Algorithm for a Data Centre Load Heliyon, 7(9):e08054

Amjady N, Rad HN (2010) Solution of nonconvex and nonsmooth economic dispatch by a new adaptive real coded genetic algorithm. Expert System Applications 37(7):5239-5245

Ang KM, Lim WH, Isa NAM, Tiang SS, Wong CH (2020) A Constrained Multi-swarm Particle Swarm Optimization without Velocity for Constrained Optimization Problems. Expert Systems with Applications 140:1-23.

Basu M (2011) Economic environmental dispatch using multi-objective differential evolution, Applied Soft Computing 11:2845-2853

Ben GN (2020) An accelerated differential evolution algorithm with new operators for multi-damage detection in plate-like structures. Applied Mathematical Modelling 80:366-383.

Betar A, Awadallah MA, Krishan MM (2020). A non-convex economic load dispatch problem with valve loading effect using a hybrid grey wolf optimizer. Neural Computing and Applications 32:12127-12154

Bhattacharya A, Chattopadhyay PK (2010) Hybrid differential evolution with biogeography-based optimization for solution of economic load dispatch. IEEE Transaction Power System 25(4):1955-1964

Bibi H, Ahmad A, Aadil F, Kim M, Muhammad K (2020) A Solution to Combined Economic Emission Dispatch (CEED) problem using Grasshopper Optimization Algorithm (GOA). International Conference on Computational Science and Computational Intelligence 712-718

Chegini SN, Bagheri A, Najafi F (2018) A new hybrid PSO based on sine cosine algorithm and Levy flight for solving optimization problems. Applied Soft Computing 73:697-726

Chen CH, Yeh SN (2006) Particle swarm optimization for economic power dispatch with valve-point effects. In: IEEE PES transmission and distribution conference and exposition Latin America, Venezuela, pp 1-5

Chen Y, Li L, Xiao J, Yang Y, Liang J, Li T (2018) Particle swarm optimizer with crossover operation. Engineering Applications of Artificial Intelligence 70:59-169

Civicioglu P (2013) Backtracking Search Optimization Algorithm for numerical optimization problems. Applied Mathematics and Computation 219:8121-8144

Coelho LDS, Mariani VC (2006) Combining of chaotic differential evolution and quadratic programming for economic dispatch optimization with valve-point effect. IEEE Transaction Power System 21(2):989-996

Coelho LDS, Mariani VC (2010) An efficient cultural self-organizing migrating strategy for economic dispatch optimization with valve-point effect. Journal Energy Conversion and Management 51(12):2580-2587

Das KN, Parouha RP (2015) An ideal tri-population approach for unconstrained optimization and applications. Applied Mathematics and Computation 256:666-701 
Dash J, Dam B, Swain R (2020) Design and implementation of sharp edge FIR filters using hybrid differential evolution particle swarm optimization. AEU - International Journal of Electronics and Communications 114:1-61

Davis L (1991) Handbook of Genetic Algorithms

Deb K (1995) Optimization for Engineering Design: Algorithms and Examples. Prentice-Hall of India, New Delhi

Devi AL, Krishna OV (2008) Combined economic and emission dispatch using evolutionary algorithms - a case study. ARPN Journal of Engineering and Applied Sciences 3(6):28-35

Edwin Selva Rex CR, Marsaline Beno M, Annrose J (2019) A Solution for Combined Economic and Emission Dispatch Problem using Hybrid Optimization Techniques. Journal of Electrical Engineering \& Technology doi.org/10.1007/s42835-019-00192-z

Eskandar H, Sadollah A, Bahreininejad A, Hamdi M (2012) Water cycle algorithm-A novel metaheuristic optimization method for solving constrained engineering optimization problems. Computers \& Structures 110-111:151-166

Espitia HE, Sofrony, JI (2018) Statistical analysis for vortex particle swarm optimization. Applied Soft Computing 67:370-386

Famelis IT, Alexandridis A, Tsitouras C (2017) A highly accurate differential evolution-particle swarm optimization algorithm for the construction of initial value problem solvers. Engineering Optimization 50(8):1364-1379

Faramarzi A, Heidarinejad M, Stephens B. Mirjalili S (2019) Equilibrium optimizer: A novel optimization algorithm. Knowledge-Based Systems 191:1-34

Gandomi AH, Alavi AH (2012) Krill herd: a new bio-inspired optimization algorithm. Communications in Nonlinear Science and Numerical Simulation 17(12):4831-4845

Geem ZW, Kim JH, Loganathan GV (2001) A new heuristic optimization algorithm: harmony search. Simulation 76(2):60-68

Goudarzi A, Li Y, Xiang J (2020) A hybrid non-linear time-varying double-weighted particle swarm optimization for solving non-convex combined environmental economic dispatch problem. Applied Soft Computing 86:1-54

Gui L, Xia X, Yu F, Wu H, Wu R, Wei B, He G (2019) A multi-role based differential evolution. Swarm and Evolutionary Computation 50:1-15

Güvenç U, Sonmez Y, Duman S, Yorükeren N (2012) Combined economic and emission dispatch solution using gravitational search algorithm. Computers \& Electrical Engineering 19(6):17541762

Hardiansyah (2013) A modified particle swarm optimization technique for economic load dispatch with valve-point effect. International Journal of Intelligent Systems and Applications 7:32-41

Hardiansyah, Junaidi, Yohannes MS (2013) An efficient simulated annealing algorithm for economic load dispatch problems. Telecommunication, Computing, Electronics and Control 11(1):37-46

Hassan BA, Rashid TA (2019a) Operational framework for recent advances in backtracking search optimisation algorithm: A systematic review and performance evaluation. Applied Mathematics and Computation https://doi.org/10.1016/j.amc.2019.124919

Hassan BA, Rashid TA (2019b) Datasets on statistical analysis and performance evaluation of backtracking search optimization algorithm compared with its counterpart algorithms. Data in brief 28:105046

Hassan MH, Kamel S, Salih SQ, Khurshaid T, Ebeed M (2021) Developing Chaotic Artificial Ecosystem-Based Optimization Algorithm for Combined Economic Emission Dispatch. In IEEE Access, 9:51146-51165, doi: 10.1109/ACCESS.2021.3066914

Heidari AA, Mirjalili S, Faris H, Aljarah I, Mafarja M, Chen H (2019) Harris hawks optimization: Algorithm and applications. Future Generation Computer Systems 97:849-872

Hosseini SA, Hajipour A, Tavakoli H (2019) Design and optimization of a CMOS power amplifier using innovative fractional-order particle swarm optimization. Applied Soft Computing 85:1-10

Hu L, Hua W, Lei W, Xiantian Z (2020) A modified Boltzmann Annealing Differential Evolution algorithm for inversion of directional resistivity logging-while-drilling measurements. Journal of Petroleum Science and Engineering 180:1-10 
Huang H, Jiang L, Yu X, Xie D (2018) Hypercube-Based Crowding Differential Evolution with Neighborhood Mutation for Multimodal Optimization. International Journal of Swarm Intelligence Research 9(2):15-27

Isiet M, Gadala M (2019) Self-adapting control parameters in particle swarm optimization. Applied Soft Computing 83:1-24

Jiang S, Zhang C, Wu W, Chen S (2019) Combined Economic and Emission Dispatch Problem of Wind-Thermal Power System Using Gravitational Particle Swarm Optimization Algorithm. Mathematical Problems in Engineering 2019:1-19.

Karaboga D, Basturk B (2007) A powerful and efficient algorithm for numerical function optimization: artificial bee colony algorithm. Journal of Global Optimization 39(3):459-471

Kennedy J, Eberhart RC (1995) Particle Swarm Optimization. In: Proceeding of IEEE International Conference on Neural Networks, pp 1942-1948

Khajeh A, Ghasemi MR, Arab HG (2019) Modified particle swarm optimization with novel population initialization. Journal of Information and Optimization Sciences 40:(6) 1167-1179

Khatsu S, Srivastava A, Das DK (2020) Solving Combined Economic Emission Dispatch for Microgrid using Time Varying Phasor Particle Swarm Optimization, 6th International Conference on Advanced Computing and Communication Systems (ICACCS) 411-415

Kumar C, Alwarsamy T (2012) Solution of economic dispatch problem using differential evolution algorithm. International Journal of Soft Computing and Engineering 1(6):236-241

Lanlan K, Ruey SC, Wenliang C, Yeh C (2020) Non-inertial opposition-based particle swarm optimization and its theoretical analysis for deep learning applications. Applied Soft Computing 88:1-10

Li S, Gu Q, Gong W, Ning B (2020) An enhanced adaptive differential evolution algorithm for parameter extraction of photovoltaic models. Energy Conversion and Management 205: 1-16

Liu ZG, Ji XH, Yang Y (2019) Hierarchical Differential Evolution Algorithm Combined with MultiCross Operation. Expert Systems with Applications 130:276-292

Mahdi FP, Vasant P, Abdullah-Al-Wadud M, Kallimani V, Watada J (2019) Quantum-behaved bat algorithm for many-objective combined economic emission dispatch problem using cubic criterion function. Neural Computing and Applications 31:5857-5869

Mahmoodabadi MJ, Mottaghi ZS, Bagheri A (2014) High Exploration Particle Swarm Optimization. Journal of Information Science 273:101-111

Mansor MH et al. (2018) A hybrid optimization technique for solving economic dispatch problem. Journal of Physics: Conf. Series 1049:1-7

Manteaw ED, Odero NA (2012) Combined economic and emission dispatch solution using ABC_PSO hybrid algorithm with valve point loading effect. International Journal of Scientific and Research Publication 2(12):1-9

Mao B, Xie Z, Wang Y, Handroos H, Wu H (2018) A Hybrid Strategy of Differential Evolution and Modified Particle Swarm Optimization for Numerical Solution of a Parallel Manipulator. Mathematical Problems in Engineering 1-9

Mirjalili S (2016) Dragonfly algorithm: a new meta-heuristic optimization technique for solving singleobjective, discrete and multi-objective problems. Neural Computing and Applications 27(4):1053-1073

Mirjalili S, Lewis A (2016) The Whale optimization algorithm. Advances in Engineering Software 95:51-67

Mirjalili S, Mirjalili SM, Lewis A (2014) Grey wolf optimizer. Advances in Engineering Software 69:46-61

Park JB, Jeong YW, Shin JR, Lee KY (2010) An improved particle swarm optimization for nonconvex economic dispatch problems. IEEE Transaction Power System 25(1):156-166

Parouha RP, Das KN (2015) An Efficient Hybrid Technique for Numerical Optimization and Applications. Computers \& Industrial Engineering 83:193-216

Parouha RP, Das KN (2016a) A Robust Memory Based Hybrid Differential Evolution for Continuous Optimization Problem. Knowledge-Based Systems 103:118-131

Parouha RP, Das KN, (2016b) An intelligent parallel hybrid algorithm for economic load dispatch problems with various practical constraints. Expert Systems with Applications 63:295-309 
Parouha RP, Verma P (2021) An innovative hybrid algorithm for bound-unconstrained optimization problems and applications. Journal of Intelligent Manufacturing doi.org/10.1007/s10845-02001691-x

Parouha RP; Verma P (2020) An innovative hybrid algorithm to solve nonconvex economic load dispatch problem with or without valve point effects. International Transactions on Electrical Energy Systems 34(1):1-67

Qiu X, Xu JX, Xu Y, Tan KC (2018) A New Differential Evolution Algorithm for Minimax Optimization in Robust Design, IEEE Transactions on Cybernetics 48(5):1355-1368.

Rao RV, Savsani VJ, Vakharia DP (2011) Teaching-learning-based optimization: a novel method for constrained mechanical design optimization problems. Computer-Aided Design 43(3):303-315

Rashedi E, Nezamabadi-pour H, Saryazdi S (2009) A Gravitational Search Algorithm. Information Sciences 179(13):2232-2248

Rashid HA, Mohammed KA, Firas MFF (2020) A New Enhancement on PSO Algorithm for Combined Economic-Emission Load Dispatch Issues. international journal of intelligent engineering \& system 13(1):77-85

Rezaie H, Kazemi-Rahbar MH, Vahidi B, Rastegar H (2018) Solution of combined economic and emission dispatch problem using a novel chaotic improved harmony search algorithm. Journal of Computational Design and Engineering 6(3):447-467

Sadollah A, Bahreininejad A, Eskandar H, Hamdi M (2013) Mine blast algorithm: a new population based algorithm for solving constrained engineering optimization problems. Applied Soft Computation 13(5):2592-2612

Sakthivel VP, Suman M, Sathya PD (2021) Combined economic and emission power dispatch problems through multi-objective squirrel search algorithm. Applied Soft Computing. 100, doi:10.1016/j.asoc.2020.106950

Secui DC (2015) A new modified artificial bee colony algorithm for the economic dispatch problem. Energy Conversion and Management 89:43-62

Serapião ABS (2009) Fundamentos de otimizaçãopor inteligência de enxames: uma visão geral. Revista SBA Controle and Automação 20(3):271-304

Serapiao ABS (2013) Cuckoo search for solving economic dispatch load problem. Intelligent Control Automat 4:385-390

Seyedmahmoudian M. et al. (2015) Simulation and hardware implementation of new maximum power point tracking technique for partially shaded PV system using hybrid DEPSO method. Transactions on Sustainable Energy 6(3):850-862

Simpson AR, Dandy GC, Murphy LJ (1994) Genetic algorithms compared to other techniques for pipe optimization. Journal of Water Resources Planning and Management 20:423-443

Storn R, Price K (1997) Differential evolution - a simple and efficient heuristic for global optimization over continuous spaces. Journal of Global Optimization 11:341-359

Subbaraj P, Rengaraj R, Salivahanan S (2011) Enhancement of Self-adaptive real coded genetic algorithm using Taguchi method for economic dispatch problem. Applied Soft Computing 11(1):83-92

Tanabe R, Fukunaga A (2013) Success-history based parameter adaptation for Differential Evolution. In IEEE Congress on Evolutionary Computation 71-78

Tang B, Xiang K, Pang M (2018) An integrated particle swarm optimization approach hybridizing a new self-adaptive particle swarm optimization with a modified differential evolution. Neural Computing and Applications 1-35

Tang B, Zhu Z, Luo J (2016) Hybridizing Particle Swarm Optimization and Differential Evolution for the Mobile Robot Global Path Planning. International Journal of Advanced Robotic Systems 13(3):1-17

Too J, Abdullah, Saad NM (2019) Hybrid Binary Particle Swarm Optimization Differential EvolutionBased AR Feature Selection for EMG Signals Classification. Axioms 8(3):1-17

Verma P, Parouha RP (2021) Non-convex Dynamic Economic Dispatch Using an Innovative Hybrid Algorithm. Journal of Electrical Engineering \& Technology doi.org/10.1007/s42835-021-00926$\mathrm{y}$ 
Wang L, Li LP (2013) An effective differential harmony search algorithm for the solving non-convex economic load dispatch problems. International Journal of Electric Power Energy System 44:832-843

Wang Y, Li B, Weise T (2010) Estimation of distribution and differential evolution cooperation for large scale economic load dispatch optimization of power systems. Information Sciences 180(12):2405-2420

Wolpert DH, Macready WG (1997) No free lunch theorems for optimization. IEEE Transactions on Evolutionary Computation 1(1):67-82

Xia X, Gui L, He G, Xie C, Wei B, Xing Y, Tang Y (2018) A hybrid optimizer based on firefly algorithm and particle swarm optimization algorithm. Journal of Computational Science 26:488500

Xiong H, Qiu B, Liu J (2020) An Improved Multi-swarm Particle Swarm Optimizer for Optimizing the Electric Field Distribution of Multichannel Transcranial Magnetic Stimulation. Artificial Intelligence In Medicine 104:1-14

Yan B, Zhao Z, Zhou Y, Yuan W, Li J, Wu J, Cheng D (2017) A Particle Swarm Optimization Algorithm with Random Learning Mechanism and Levy Flight for Optimization of Atomic Clusters. Computer Physics Communication 219:79-86

Yang X, Li J, Peng X (2019) An improved differential evolution algorithm for learning high-fidelity quantum controls. Science Bulletin 64(19):1402-1408

Yang XS, Deb S (2009) Cuckoo Search via Lévy flights. In proceedings of World Congress on Nature \& Biologically Inspired Computing, Coimbatore, India, pp 210-214

Yu H, Tan Y, Zeng J, Sun C, Jin Y (2018) Surrogate-assisted hierarchical particle swarm optimization. Information Sciences 454-455:59-72

Zhang H, Li X (2018) Enhanced differential evolution with modified parent selection technique for numerical optimization. International Journal of Computational Science and Engineering 17(1): 98-108

Zhang J, Sanderson C (2009) JADE: Adaptive Differential Evolution with optional external archive. IEEE Transactions on Evolutionary Computation 13(5):945-958

Zhang R, Zhou J, Mo L, Ouyang S, Liao X (2013) Economic environmental dispatch using an enhanced multi-objective cultural algorithm. Electric Power Systems Research 99:18-29 